PKU-TP-98-53

\title{
Diffractive light quark jet and gluon jet production at hadron colliders in the two-gluon exchange model
}

\author{
Feng Yuan \\ Department of Physics, Peking University, Beijing 100871, People's Republic of China \\ Kuang-Ta Chao \\ China Center of Advanced Science and Technology (World Laboratory), Beijing 100080, People's \\ Republic of China \\ and Department of Physics, Peking University, Beijing 100871, People's Republic of China
}

\begin{abstract}
Massless diquark jet and gluon jet productions at large transverse momentum in the coherent diffractive processes at hadron colliders are studied in the two-gluon exchange parametrization of the Pomeron model. We use the helicity amplitude method to calculate the cross section formulas. We find that for the light quark jet and gluon jet production the diffractive process is related to the differential off-diagonal gluon distribution function in the proton. We estimate the production rate for these processes at the Fermilab Tevatron by approximating the off-diagonal gluon distribution function by the usual diagonal gluon distribution in the proton. We also use the helicity amplitude method to calculate the diffractive charm jet production at hadron colliders, by which we reproduce the leading logarithmic approximation result of this process we previously calculated.
\end{abstract}

PACS number(s): 12.40.Nn, 13.85.Ni, 14.40.Gx 


\section{INTRODUCTION}

In recent years, there has been a renaissance of interest in diffractive scattering. These diffractive processes are described by the Regge theory in terms of the Pomeron $(\mathbb{P})$ exchange [1]. The Pomeron carries quantum numbers of the vacuum, so it is a colorless entity in QCD language, which may lead to the "rapidity gap" events in experiments. However, the nature of Pomeron and its reaction with hadrons remain a mystery. For a long time it had been understood that the dynamics of the "soft pomeron" is deeply tied to confinement. However, it has been realized now that how much can be learned about QCD from the wide variety of small- $x$ and hard diffractive processes, which are now under study experimentally. In Refs. [2,3], the diffractive $J / \psi$ and $\Upsilon$ production cross section have been formulated in photoproduction processes and in DIS processes in perturbative QCD. In the framework of perturbative QCD the Pomeron is represented by a pair of gluon in the color-singlet sate. This two-gluon exchange model can successfully describe the experimental results from HERA [5].

On the other hand, as we know that there exist nonfactorization effects in the hard diffractive processes at hadron colliders [6 9]. First, there is the so-called spectator effect 8], which can change the probability of the diffractive hadron emerging from collisions intact. Practically, a suppression factor (or survive factor) " $S_{F}$ " is used to describe this effect [10]. Obviously, this suppression factor can not be calculated in perturbative QCD, which is now viewed as a nonperturbative parameter. Typically, the suppression factor $S_{F}$ is determined to be about 0.1 at the energy scale of the Fermilab Tevatron [9]. Another nonfactorization effect discussed in literature is associated with the coherent diffractive processes at hadron colliders [7], in which the whole Pomeron is induced in the hard scattering. It is proved in [7] that the existence of the leading twist coherent diffractive processes is associated with a breakdown of the QCD factorization theorem.

Based on the success of the two-gluon exchange parametrization of the Pomeron model in the description of the diffractive photoproduction processes at $e p$ colliders [2, 3, 5], we may extend the applications of this model to calculate the diffractive processes at hadron colliders in perturbative QCD. Under this context, the Pomeron represented by a color-singlet twogluon system emits from one hadron and interacts with another hadron in hard process, in which the two gluons are both involved (see Fig. 1). Therefore, these processes calculated in the two-gluon exchange model are just belong to the coherent diffractive processes in hadron collisions. Another important feature of the calculations of the diffractive processes in this model recently demonstrated is the sensitivity to the off-diagonal parton distribution function in the proton [11].

Using this two-gluon exchange model, we have calculated the diffractive $J / \psi$ production [12], charm jet production [13], massive muon pair and $W$ boson productions [14] in hadron collisions. These calculations show that we can explore the off-diagonal gluon distribution function in low $x$ region and study the coherent diffractive processes at hadron colliders through these processes. In this paper, we will calculate the light quark (massless) jet and gluon jet productions at large transverse momentum in the coherent diffractive processes at hadron colliders by using the two-gluon exchange model. As sketched in Fig. 1, the two-gluon system (in color-singlet) emitted from one hadron interacts with another hadron to produce

the final state light quark (or gluon) jets. There are three different partonic processes 
contributing to the diffractive dijet productions, $g p \rightarrow q \bar{q} p, q p \rightarrow q g p$, and $g p \rightarrow g g p$ as shown in Fig.2, Fig.3 and Fig.4 respectively.

In the calculations of Refs. [12 14], there always is a large mass scale associated with the production process. That is $M_{\psi}$ for $J / \psi$ production, $m_{c}$ for the charm jet production, $M^{2}$ for the massive muon production $\left(M^{2}\right.$ is the invariant mass of the muon pair) and $M_{W}^{2}$ for $W$ boson production. However, in the processes we will calculate in this paper, there is no large mass scale. So, for the light quark jet and gluon jet production, the large transverse momentum is needed to guarantee the application of the perturbative QCD. Furthermore, the experience on the calculations of the diffractive di-quark jet photoproduction [15] shows that the light quark jet production in the two-gluon exchange model has a distinctive feature that there is no contribution from the small $l_{T}^{2}$ region $\left(l_{T}^{2}<k_{T}^{2}\right)$ in the integration of the amplitude over $l_{T}^{2}$. So, the expansion (in terms of $l_{T}^{2} / M_{X}^{2}$ ) method used in Refs. [12 14 can not be applied for the calculations here. In the following calculations, we will employ the helicity amplitude method to calculate the amplitude of the diffractive light quark jet and gluon jet production in hadron collisions. We will show that the production cross sections for these processes are related to the differential (off-diagonal) gluon distribution function in the proton as that in the diffractive di-quark jet photoprodution process [15]. (In contrast, we note that the cross sections of the processes calculated in Refs. [12 114 are related to the integrated gluon distribution function in the proton).

The diffractive production of heavy quark jet at hadron colliders has also been studied by using the two-gluon exchange model in Ref. [16]. However, their approach is very different from ours 1 . In their calculations, they separated their diagrams into two parts, and called one part the coherent diffractive contribution to the heavy quark production. In our approach, in line with the definition of Ref. [7], we call the process in which the whole Pomeron participates in the hard scattering process as the coherent diffractive process. Under this definition, all of the diagrams plotted in Fig.2, Fig.3 and Fig.4 for the partonic processes $g p \rightarrow q \bar{q} p, q p \rightarrow q g p$ and $g p \rightarrow g g p$ contribute to the coherent diffractive production.

In addition, we must emphasize that in our calculations we only discuss the contribution to the diffractive dijet production from the so-called "Pomeron fragmentation" region, i.e., $M_{X}^{2} \sim 4 k_{T}^{2}$, where $M_{X}^{2}$ is the invariant mass of the diffractive final states and $k_{T}$ is the transverse momenta of the outgoing jet. However, in the region of $M_{X}^{2} \gg 4 k_{T}^{2}$, the diffractive production may be dominated by the central (for $M_{X}^{2}$ system) or initial hadron fragmentation contributions, and in this region a resolved Pomeron model (with parton distributions for the Pomeron) may be more appropriate.

The rest of the paper is organized as follows. In Sec.II, we will give the cross section formulas for the partonic processes of Figs.2-4 in the leading order of perturbative QCD, where we employ the helicity amplitude method to calculate the amplitude for these processes. We will also use this method to recalculate the diffractive charm jet production process $g p \rightarrow c \bar{c} p$, by which we can reproduce the leading logarithmic approximation result given in Ref. [13]. In Sec.III, the numerical results for the diffractive light quark jet and gluon jet production at the Fermilab Tevatron will be given. And the conclusions will be given in Sec.IV.

\footnotetext{
${ }^{1}$ For detailed discussions and comments, please see 13
} 


\section{THE CROSS SECTION FORMULAS FOR THE PARTONIC PROCESSES}

In the leading order of perturbative QCD, the Feynman diagrams for the three different partonic processes are plotted in Figs. 2-4 respectively. The two-gluon system coupled to the proton (antiproton) in these diagrams is in a color-singlet state, which characterizes the diffractive processes in perturbative QCD. Due to the positive signature of these diagrams (color-singlet exchange), we know that the real part of the amplitude cancels out in the leading logarithmic approximation. To get the imaginary part of the amplitude, we must calculate the discontinuity represented by the crosses in each diagram of these figures.

In our calculations, we express the formulas in terms of the Sudakov variables. That is, every four-momenta $k_{i}$ are decomposed as,

$$
k_{i}=\alpha_{i} q+\beta_{i} p+\vec{k}_{i T}
$$

where $q$ and $p$ are the momenta of the incident parton (quark or gluon) and the diffractive proton, $q^{2}=0, p^{2}=0$, and $2 p \cdot q=W^{2}=s . \alpha_{i}$ and $\beta_{i}$ are the momentum fractions of $q$ and $p$ respectively. $k_{i T}$ is the transverse momentum, which satisfies

$$
k_{i T} \cdot q=0, \quad k_{i T} \cdot p=0
$$

All of the Sudakov variables for every momentum are determined by using the on-shell conditions of the momenta represented by the external lines and the crossed lines in the diagram. The calculations of these Sudakov variables are similar to those in the diffractive charm jet production process $g p \rightarrow c \bar{c} p$ [13].

For the momentum $u$, we have

$$
\alpha_{u}=0, \quad \beta_{u}=x_{I P}=\frac{M_{X}^{2}}{s}, \quad u_{T}^{2}=t=0
$$

where $M_{X}^{2}$ is the invariant mass squared of the diffractive final state. For the high energy diffractive process, we know that $M_{X}^{2} \ll s$, so we have $\beta_{u}\left(x_{I P}\right)$ as a small parameter. For the momentum $k$,

$$
\alpha_{k}\left(1+\alpha_{k}\right)=-\frac{k_{T}^{2}}{M_{X}^{2}}, \quad \beta_{k}=-\alpha_{k} \beta_{u}
$$

where $k_{T}$ is the transverse momentum of the out going quark jet (or gluon jet).

For the loop mentum $l$, the Sudakov parameters are not the same for every diagrams and every partonic processes, so we will discuss them in the following subsections respectively.

By the form of these Sudakov variables, the cross section formula for the partonic process can be formulated as

$$
\left.\frac{d \hat{\sigma}}{d t}\right|_{t=0}=\frac{d M_{X}^{2} d^{2} k_{T} d \alpha_{k}}{16 \pi s^{2} 16 \pi^{3} M_{X}^{2}} \delta\left(\alpha_{k}\left(1+\alpha_{k}\right)+\frac{k_{T}^{2}}{M_{X}^{2}}\right) \sum \overline{\mid \mathcal{A}}^{2} .
$$

$\mathcal{A}$ is the amplitude of the partonic process, and we know that the real part of the amplitude is zero in the leading order. So, we only need to calculate the imaginary parts of the amplitudes for the three partonic processes. 
In the calculations of the amplitudes for the three partonic processes, we employ the Helicity Amplitude method 17 19. As a cross check, we will recalculate the diffractive charm jet production [13] by using this method.

Another important check to our calculations is performed by examining the behavior of the amplitude under the limit of $l_{T}^{2} \rightarrow 0$ in the integration over the loop momentum $l$. From the experience of the calculations for the diffractive charm jet production in Ref. [13], we know that the contribution to the amplitude from the individual diagram of the parton process has linear singularity at $l_{T}^{2} \rightarrow 0$. The linear singularity is not proper in QCD perturbative calculations. So, the linear singularities coming from different diagrams must be canceled out by each other for the same order of perturbative calculations. That is to say we must guarantee the total sum of the contributions from all of the diagrams free of the linear singularity in the calculations for every partonic processes.

\section{A. $g p \rightarrow q \bar{q} p$ process}

The first four diagrams of Fig. 2 for this subprocess are the same as those calculated in the diffractive photoproduction processes 2 2 [4]. But, due to the existence of gluon-gluon interaction vertex in $\mathrm{QCD}$, in the partonic process $g p \rightarrow q \bar{q} p$, there are additional five diagrams (Diags. (5)-(9)). These five diagrams are needed for complete calculations in this order of QCD.

First, we give the Sudakov parameters for the loop mentum $l$ for this process (Fig. 2),

$$
\begin{aligned}
\alpha_{l} & =-\frac{l_{T}^{2}}{s}, \\
\beta_{l} & =\frac{2\left(k_{T}, l_{T}\right)-l_{T}^{2}}{\alpha_{k} s}, \quad \text { for Diag.1, 3, 5, } \\
& =\frac{2\left(k_{T}, l_{T}\right)+l_{T}^{2}}{\left(1+\alpha_{k}\right) s}, \quad \text { for Diag.2, 4, 6, } \\
& =-\frac{M_{X}^{2}-l_{T}^{2}}{s}, \quad \text { for Diag.7, 8, 9, }
\end{aligned}
$$

where $\left(k_{T}, l_{T}\right)$ is the 2-dimensional product of the transverse vectors $\vec{k}_{T}$ and $\vec{l}_{T}$.

The imaginary part of the amplitude $\mathcal{A}(g p \rightarrow q \bar{q} p)$ for each diagram of Fig. 2 has the following general form,

$$
\operatorname{Im} \mathcal{A}=C_{F}\left(T_{i j}^{a}\right) \int \frac{d^{2} l_{T}}{\left(l_{T}^{2}\right)^{2}} F \times \bar{u}_{i}(k+q) \Gamma_{\mu} v_{j}(u-k),
$$

where $C_{F}$ is the color factor for each diagram, and is the same as that for the $g p \rightarrow c \bar{c} p$ process [13]. $a$ is the color index of the incident gluon. $\Gamma_{\mu}$ represents some $\gamma$ matrices including one propagator. $F$ in the integral is defined as

$$
F=\frac{3}{2 s} g_{s}^{3} f\left(x^{\prime}, x^{\prime \prime} ; l_{T}^{2}\right)
$$

where 


$$
f\left(x^{\prime}, x^{\prime \prime} ; l_{T}^{2}\right)=\frac{\partial G\left(x^{\prime}, x^{\prime \prime} ; l_{T}^{2}\right)}{\partial \ln l_{T}^{2}},
$$

where the function $G\left(x^{\prime}, x^{\prime \prime} ; k_{T}^{2}\right)$ is the so-called off-diagonal gluon distribution function [11. Here, $x^{\prime}$ and $x^{\prime \prime}$ are the momentum fractions of the proton carried by the two gluons. It is expected that at small $x$, there is no big difference between the off-diagonal and the usual diagonal gluon densities [20]. So, in the following calculations, we estimate the production rate by approximating the off-diagonal gluon density by the usual diagonal gluon density, $G\left(x^{\prime}, x^{\prime \prime} ; Q^{2}\right) \approx x g\left(x, Q^{2}\right)$, where $x=x_{\mathbb{P}}=M_{X}^{2} / s$.

In Ref. [13], we calculate the amplitude (7) for the heavy quark diffractive production processes by expanding $\Gamma_{\mu}$ in terms of $l_{T}^{2}$. However, in the light quark jet production process $g p \rightarrow q \bar{q} p$, the expansion method is not yet valid. According to the result of [13], the production cross section is proportional to the heavy quark mass. If we apply this formula to the light quark jet production, the cross section will be zero. That is to say, the expansion of the amplitude in terms of $l_{T}^{2}$, in which the large logarithmic contribution comes from the region of $l_{T}^{2} \ll M_{X}^{2}$, is not further suitable for the calculation of the cross section for massless light quark jet production. Furthermore, the experience of the calculation of the diffractive light quark jet photoproduction process $\gamma p \rightarrow q \bar{q} p\left(\right.$ for $\left.Q^{2}=0\right)$ [15] indicates that there is no contribution from the region of $l_{T}^{2}<k_{T}^{2}$ in the integration of the amplitude over $l_{T}^{2}$. In the hadroproduction process $g p \rightarrow q \bar{q} p$, the situation is the same. So, the expansion method, in which $l_{T}^{2}$ is taken as a small parameter, is not valid for the calculations of the massless quark production processes.

In the following, we employ the helicity amplitude method [19] to calculate the amplitude Eq.(7). For the massless quark spinors, we define

$$
u_{ \pm}(p)=\frac{1}{\sqrt{2}}\left(1 \pm \gamma_{5}\right) u(p)
$$

For the polarization vector of the incident gluon, which is transversely polarized, we choose,

$$
e_{ \pm}=\frac{1}{\sqrt{2}}(0,1, \pm i, 0)
$$

The helicity amplitudes for the processes in which the polarized Dirac particles are involved have the following general forms [19],

$$
\bar{u}_{ \pm}\left(p_{f}\right) Q v_{\mp}\left(p_{i}\right)=\frac{\operatorname{Tr}\left[Q \not p_{i} \not h \not p_{f}\left(1 \mp \gamma_{5}\right)\right]}{4 \sqrt{\left(n \cdot p_{i}\right)\left(n \cdot p_{f}\right)}}
$$

where $n$ is an arbitrary massless 4 -vector, which is set to be $n=p$ in the following calculations. Using this formula (12), the calculations of the helicity amplitude $\mathcal{A}(\lambda(g), \lambda(q), \lambda(\bar{q}))$ for the diffractive process $g p \rightarrow q \bar{q} p$ is straightforward. Here $\lambda$ are the corresponding helicities of the external gluon, quark and antiquark. In our calculations, we only take the leading order contribution, and neglect the higher order contributions which are proportional to $\beta_{u}=\frac{M_{X}^{2}}{s}$ because in the high energy diffractive processes we have $\beta_{u} \ll 1$.

For the first four diagrams, to sum up together, the imaginary part of the amplitude $\mathcal{A}( \pm,+,-)$ is 


$$
\operatorname{Im} \mathcal{A}^{1234}( \pm,+,-)=\alpha_{k}^{2}\left(1+\alpha_{k}\right) \mathcal{N} \times \int \frac{d^{2} \vec{l}_{T}}{\left(l_{T}^{2}\right)^{2}} f\left(x^{\prime}, x^{\prime \prime} ; l_{T}^{2}\right)\left(-\frac{2}{9} \frac{\vec{e}^{( \pm)} \cdot \vec{k}_{T}}{k_{T}^{2}}-\frac{1}{36} \frac{\vec{e}^{( \pm)} \cdot\left(\vec{k}_{T}-\vec{l}_{T}\right)}{\left(\vec{k}_{T}-\vec{l}_{T}\right)^{2}}\right)
$$

where $\frac{2}{9}$ and $-\frac{1}{36}$ are the color factors for Diag.1,4 and Diag.2,3 respectively, and $\mathcal{N}$ is defined as

$$
\mathcal{N}=\frac{s}{\sqrt{-\alpha_{k}\left(1+\alpha_{k}\right)}} g_{s}^{3} T_{i j}^{a} .
$$

The other helicity amplitudes for the first four diagrams have the similar form as (13). The amplitude expression Eq. (13) is the same as that for the photoproduction process $\gamma p \rightarrow q \bar{q} p$ previously calculated in Refs. [4,15] except the difference on the color factors. In the diffractive photoproduction process, the color factors of these four diagrams are the same (they are all $\frac{2}{9}$ ), while in hadroproduction process the color factors are no longer the same for these four diagrams. It is instructive to see what is the consequence of this difference. We know that the amplitude of the diffractive process in Eq. (7) must be zero in the limit $l_{T}^{2} \rightarrow 0$. Otherwise, this will lead to a linear singularity when we perform the integration of the amplitude over $l_{T}^{2}$ due to existence of the factor $1 /\left(l_{T}^{2}\right)^{2}$ in the integral of Eq. (7) [13]. This linear singularity is not proper in QCD calculations. So, we must first exam the amplitude behavior under the limit of $l_{T}^{2} \rightarrow 0$ for all the diffractive processes in the calculations using the two-gluon exchange model. From Eq. (13), we can see that the amplitude for the diffractive photoproduction of di-quark jet process is exact zero at $l_{T}^{2} \rightarrow 0$. However, for the diffractive hadroproduction process $g p \rightarrow q \bar{q} p$ the amplitude for the first four diagrams is not exact zero in the limit $l_{T}^{2} \rightarrow 0$ due to the inequality of the color factors between them. So, for $g p \rightarrow q \bar{q} p$ process there must be other diagrams in this order of perturbative QCD calculation to cancel out the linear singularity which rises from the first four diagrams. The last five diagrams of Fig.2 are just for this purpose.

For example, the contributions from Diags. 5 and 8 are

$$
\operatorname{Im} \mathcal{A}^{58}( \pm,+,-)=\alpha_{k}^{2}\left(1+\alpha_{k}\right) \mathcal{N} \times \int \frac{d^{2} \vec{l}_{T}}{\left(l_{T}^{2}\right)^{2}} f\left(x^{\prime}, x^{\prime \prime} ; l_{T}^{2}\right)\left(-\frac{1+\alpha_{k}}{4} \frac{\vec{e}^{( \pm)} \cdot\left(\vec{k}_{T}-\left(1+\alpha_{k}\right) \vec{l}_{T}\right)}{\left(\vec{k}_{T}-\left(1+\alpha_{k}\right)^{2} \vec{l}_{T}\right)^{2}}\right)
$$

and the contributions from Diags.6 and 9 are

$$
\operatorname{Im} \mathcal{A}^{69}( \pm,+,-)=\alpha_{k}^{2}\left(1+\alpha_{k}\right) \mathcal{N} \times \int \frac{d^{2} \vec{l}_{T}}{\left(l_{T}^{2}\right)^{2}} f\left(x^{\prime}, x^{\prime \prime} ; l_{T}^{2}\right)\left(\frac{\alpha_{k}}{4} \frac{\vec{e}^{( \pm)} \cdot\left(\vec{k}_{T}-\alpha_{k} \vec{l}_{T}\right)}{\left(\vec{k}_{T}-\alpha_{k}^{2} \vec{l}_{T}\right)^{2}}\right),
$$

and the contribution from Diag.7 is

$$
\operatorname{Im} \mathcal{A}^{7}( \pm,+,-)=\alpha_{k}^{2}\left(1+\alpha_{k}\right) \mathcal{N} \times \int \frac{d^{2} \vec{l}_{T}}{\left(l_{T}^{2}\right)^{2}} f\left(x^{\prime}, x^{\prime \prime} ; l_{T}^{2}\right)\left(\frac{1}{2} \frac{e^{-( \pm)} \cdot \vec{k}_{T}}{k_{T}^{2}}\right)
$$

From the above results, we can see that the contributions from Diags.5-9 just cancel out the linear singularity which rises from the first four diagrams. Their total sum from the nine diagrams of Fig.2 is free of linear singularity now. 
Finally, by adding up all of the nine diagrams of Fig.2, the imaginary parts of the amplitudes for the following helicity sets are,

$$
\begin{aligned}
& \operatorname{Im} \mathcal{A}( \pm,+,-)=\alpha_{k}^{2}\left(1+\alpha_{k}\right) \mathcal{N} \times \mathcal{T}^{( \pm)} \\
& \operatorname{Im} \mathcal{A}( \pm,-,+)=\alpha_{k}\left(1+\alpha_{k}\right)^{2} \mathcal{N} \times \mathcal{T}^{( \pm)}
\end{aligned}
$$

where

$$
\begin{aligned}
\mathcal{T}^{( \pm)}= & \int \frac{d^{2} \vec{l}_{T}}{\left(l_{T}^{2}\right)^{2}} f\left(x^{\prime}, x^{\prime \prime} ; l_{T}^{2}\right)\left[\left(\frac{1}{2}-\frac{2}{9}\right) \frac{e^{( \pm)} \cdot \vec{k}_{T}}{k_{T}^{2}}-\frac{1}{36} \frac{e^{( \pm)} \cdot\left(\vec{k}_{T}-\vec{l}_{T}\right)}{\left(\vec{k}_{T}-\vec{l}_{T}\right)^{2}}\right. \\
& \left.-\frac{1+\alpha_{k}}{4} \frac{\vec{e}^{( \pm)} \cdot\left(\vec{k}_{T}-\left(1+\alpha_{k}\right) \vec{l}_{T}\right)}{\left(\vec{k}_{T}-\left(1+\alpha_{k}\right) \vec{l}_{T}\right)^{2}}+\frac{\alpha_{k}}{4} \frac{\vec{e}^{( \pm)} \cdot\left(\vec{k}_{T}-\alpha_{k} \vec{l}_{T}\right)}{\left(\vec{k}_{T}-\alpha_{k} \vec{l}_{T}\right)^{2}}\right] .
\end{aligned}
$$

And the amplitudes for the other helicity sets are zero in the light quark jet production process. From the above results, we can see that in the integration of the amplitude the linear singularity from different diagrams are canceled out by each other, which will guarantee there is no linear singularity in the total sum.

Another feature of the above results for the amplitudes is the relation to the differential off-diagonal gluon distribution function $f\left(x^{\prime}, x^{\prime \prime} ; l_{T}^{2}\right)$. To get the cross section for the diffractive process, we must perform the integration of Eq. (19). However, as mentioned above that there is no big difference between the off-diagonal gluon distribution function and the usual gluon distribution at small $x$, so we can simplify the integration of (19) by approximating the differential off-diagonal gluon distribution function $f\left(x^{\prime}, x^{\prime \prime} ; l_{T}^{2}\right)$ by the usual diagonal differential gluon distribution function $f_{g}\left(x ; l_{T}^{2}\right)$.

After integrating over the azimuth angle of $\vec{l}_{T}$, the integration $\mathcal{T}^{( \pm)}$will then be

$$
\mathcal{T}^{( \pm)}=\pi \frac{\vec{e}^{( \pm)} \cdot \vec{k}_{T}}{k_{T}^{2}} \mathcal{I}
$$

where

$$
\begin{aligned}
\mathcal{I}= & \int \frac{d l_{T}^{2}}{\left(l_{T}^{2}\right)^{2}} f_{g}\left(x ; l_{T}^{2}\right)\left[\frac{1}{36}\left(\frac{1}{2}-\frac{k_{T}^{2}-l_{T}^{2}}{2\left|k_{T}^{2}-l_{T}^{2}\right|}\right)+\frac{1+\alpha_{k}}{4}\left(\frac{1}{2}-\frac{k_{T}^{2}-\left(1+\alpha_{k}\right) l_{T}^{2}}{2\left|k_{T}^{2}-\left(1+\alpha_{k}\right) l_{T}^{2}\right|}\right)\right. \\
& \left.-\frac{\alpha_{k}}{4}\left(\frac{1}{2}-\frac{k_{T}^{2}-\alpha_{k} l_{T}^{2}}{2\left|k_{T}^{2}-\alpha_{k} l_{T}^{2}\right|}\right)\right] .
\end{aligned}
$$

Comparing the above results with those of the photoproduction process $\gamma p \rightarrow q \bar{q} p$ [4, 15], we find that the amplitude formula for the diffractive light quark jet hadroproduction process $g p \rightarrow q \bar{q} p$ is much more complicated. However, the basic structure of the amplitude, especially the expression for the integration $\mathcal{I}$ is similar to that for the photoproduction process. In the integration of (21), if $l_{T}^{2}<k_{T}^{2}$ the first term of the integration over $l_{T}^{2}$ will be zero; if $l_{T}^{2}<k_{T}^{2} /\left(1+\alpha_{k}\right)^{2}$ the second term will be zero; if $l_{T}^{2}<k_{T}^{2} / \alpha_{k}^{2}$ the third term will be zero. So, the dominant regions contributing to the three integration terms are $l_{T}^{2} \sim k_{T}^{2}$, $l_{T}^{2} \sim k_{T}^{2} /\left(1+\alpha_{k}\right)^{2}$, and $l_{T}^{2} \sim k_{T}^{2} / \alpha_{k}^{2}$ respectively. Approximately, by ignoring some evolution effects of the differential gluon distribution function $f_{g}\left(x ; l_{T}^{2}\right)$ in the above dominant integration regions, we get the following results for the integration $\mathcal{I}$, 


$$
\mathcal{I}=\frac{1}{k_{T}^{2}}\left[\frac{1}{36} f_{g}\left(x ; k_{T}^{2}\right)+\frac{\left(1+\alpha_{k}\right)^{3}}{4} f_{g}\left(x ; \frac{k_{T}^{2}}{\left(1+\alpha_{k}\right)^{2}}\right)-\frac{\alpha_{k}^{3}}{4} f_{g}\left(x ; \frac{k_{T}^{2}}{\alpha_{k}^{2}}\right)\right] .
$$

Obtained the formula for the integration $\mathcal{I}$, the amplitude squared for the partonic process $g p \rightarrow q \bar{q} p$ will be reduced to, after averaging over the spin and color degrees of freedom,

$$
\overline{\mid \mathcal{A}}^{2}=\frac{9}{4} \alpha_{s}^{3}(4 \pi)^{3} \pi^{2} s^{2} \frac{|\mathcal{I}|^{2}}{M_{X}^{2}}\left(1-\frac{2 k_{T}^{2}}{M_{X}^{2}}\right) .
$$

And the cross section for the partonic process $g p \rightarrow q \bar{q} p$ is

$$
\left.\frac{d \hat{\sigma}(g p \rightarrow q \bar{q} p)}{d t}\right|_{t=0}=\int_{M_{X}^{2}>4 k_{T}^{2}} d M_{X}^{2} d k_{T}^{2} \frac{9 \alpha_{s}^{3} \pi^{2}}{8\left(M_{X}^{2}\right)^{2}} \frac{1}{\sqrt{1-\frac{4 k_{T}^{2}}{M_{X}^{2}}}}\left(1-\frac{2 k_{T}^{2}}{M_{X}^{2}}\right)|\mathcal{I}|^{2} .
$$

The integral bound $M_{X}^{2}>4 k_{T}^{2}$ above shows that the dominant contribution of the integration over $M_{X}^{2}$ comes from the region of $M_{X}^{2} \sim 4 k_{T}^{2}$. Using Eq. (四), this indicates that in this dominant region $\alpha_{k}$ is of order of 1 . So, in the integration $\mathcal{I}$ the differential gluon distribution function $f_{g}\left(x ; Q^{2}\right)$ of the three terms can approximately take their values at the same scale of $Q^{2}=k_{T}^{2}$. That is, the integration $\mathcal{I}$ is then simplified to

$$
\mathcal{I} \approx \frac{10 M_{X}^{2}-27 k_{T}^{2}}{36 M_{X}^{2}} f_{g}\left(x ; k_{T}^{2}\right) .
$$

Numerical calculations show that there is little difference (within $10 \%$ for $k_{T}>5 \mathrm{GeV}$ ) between the cross sections by using these two different parametrizations of $\mathcal{I}$, Eq. (22) and Eq. (25). So, in Sec.III, we use Eqs. (24) and (25) to estimate the diffractive light quark jet production rate at the Fermilab Tevatron.

\section{B. Recalculate the heavy quark jet production using the helicity amplitude method}

For a crossing check, in this subsection we will recalculate the diffractive heavy quark jet production at hadron colliders by using the helicity amplitude method. In Ref. [13], we have calculated this process in the leading logarithmic approximation of QCD, where we expanded the amplitude in terms of $l_{T}^{2}$. Now, if we use the helicity amplitude method, we donot need to use the expansion method for the $\Gamma_{\mu}$ factor in Eq. (7) as in [13]. We can firstly calculate the amplitude explicitly by using the helicity amplitude method.

However, for the massive fermion, the amplitude formula is more complicated. Following Ref. [18], we first define the basic spinors $u_{ \pm}\left(k_{0}\right)$ as,

$$
\begin{array}{r}
u_{+}\left(k_{0}\right)=\not k_{1} u_{-}\left(k_{0}\right), \\
u_{-}\left(k_{0}\right) \bar{u}_{-}\left(k_{0}\right)=\frac{1}{2}\left(1-\gamma_{5}\right) \not k_{0},
\end{array}
$$

where the momenta $k_{0}$ and $k_{1}$ satisfy the following relations,

$$
k_{0} \cdot k_{0}=0, \quad k_{1} \cdot k_{1}=-1, \quad k_{0} \cdot k_{1}=0 .
$$


Using Eqs. (26) and (27), we can easily find that the spinor $u_{+}\left(k_{0}\right)$ satisfies

$$
u_{+}\left(k_{0}\right) \bar{u}_{+}\left(k_{0}\right)=\frac{1}{2}\left(1+\gamma_{5}\right) \not k_{0} .
$$

Provided the basic spinors, we then express any spinors $u\left(p_{i}\right)$ in terms of the basic ones, 18

$$
u_{ \pm}\left(p_{i}\right)=\frac{\left(\not p_{i}+m_{i}\right) u_{ \pm}\left(k_{0}\right)}{\sqrt{2 p_{i} \cdot k_{0}}} .
$$

It is easily checked that these spinors satisfy Dirac's equations. Now, for the massive fermions, the helicity amplitudes for the processes involving Dirac particles have the following general forms,

$$
\begin{aligned}
& \bar{u}_{+}\left(p_{f}\right) Q v_{-}\left(p_{i}\right)=\frac{\operatorname{Tr}\left[Q\left(\not p_{i}-m_{i}\right)\left(1-\gamma_{5}\right) \not k_{0}\left(\not p_{f}+m_{f}\right)\right]}{4 \sqrt{\left(k_{0} \cdot p_{i}\right)\left(k_{0} \cdot p_{f}\right)}}, \\
& \bar{u}_{-}\left(p_{f}\right) Q v_{+}\left(p_{i}\right)=\frac{\operatorname{Tr}\left[Q\left(\not p_{i}-m_{i}\right)\left(1+\gamma_{5}\right) \not k_{0}\left(\not p_{f}+m_{f}\right)\right]}{4 \sqrt{\left(k_{0} \cdot p_{i}\right)\left(k_{0} \cdot p_{f}\right)}}, \\
& \bar{u}_{+}\left(p_{f}\right) Q v_{+}\left(p_{i}\right)=\frac{\operatorname{Tr}\left[Q\left(\not p_{i}-m_{i}\right)\left(1-\gamma_{5}\right) \not \ell_{1} \not k_{0}\left(\not p_{f}+m_{f}\right)\right]}{4 \sqrt{\left(k_{0} \cdot p_{i}\right)\left(k_{0} \cdot p_{f}\right)}}, \\
& \bar{u}_{-}\left(p_{f}\right) Q v_{-}\left(p_{i}\right)=\frac{\operatorname{Tr}\left[Q\left(\not p_{i}-m_{i}\right) \not k_{1}\left(1-\gamma_{5}\right) \not k_{0}\left(\not p_{f}+m_{f}\right)\right]}{4 \sqrt{\left(k_{0} \cdot p_{i}\right)\left(k_{0} \cdot p_{f}\right)}},
\end{aligned}
$$

where $m_{i}$ and $m_{f}$ are the masses for the momenta $p_{i}$ and $p_{f}$ respectively, where $p_{i}^{2}=m_{i}^{2}, p_{f}^{2}=$ $m_{f}^{2}$. From the above equations, we can see that for the massless fermions $\left(m_{i}=m_{f}=0\right)$ the formula Eq. (30) will then turn back to the formula Eq. (12).

To calculate the imaginary part of the amplitude Eq. (17) for the partonic process $g p \rightarrow$ $c \bar{c} p$, a convenient choice for the momenta $k_{0}$ and $k_{1}$ is,

$$
k_{0}=p, \quad k_{1}=e,
$$

where the vector $e$ is the polarization vector for the incident gluon defined in Eq. (11). Using the formula Eq. (30) and the above choice for the momenta $k_{0}$ and $k_{1}$, the helicity amplitudes for Eq. (7) will then be,

$$
\begin{aligned}
& \operatorname{Im} \mathcal{A}( \pm,+,-)=\alpha_{k}^{2}\left(1+\alpha_{k}\right) \mathcal{N} \times \mathcal{T}_{c}^{( \pm)} \\
& \operatorname{Im} \mathcal{A}( \pm,-,+)=\alpha_{k}\left(1+\alpha_{k}\right)^{2} \mathcal{N} \times \mathcal{T}_{c}^{( \pm)} \\
& \operatorname{Im} \mathcal{A}( \pm,+,+)=\operatorname{Im} \mathcal{A}( \pm,-,-)=\alpha_{k}\left(1+\alpha_{k}\right) \mathcal{N} \times \frac{\pi m_{c}}{2} \mathcal{I}_{c}^{\prime}
\end{aligned}
$$

where $\mathcal{N}$ is the same as in Eq. (14), and the integrations $\mathcal{T}_{c}^{( \pm)}$and $\mathcal{I}_{c}^{\prime}$ are defined as

$$
\begin{aligned}
\mathcal{T}_{c}^{( \pm)}= & \int \frac{d^{2} \vec{l}_{T}}{\left(l_{T}^{2}\right)^{2}} f\left(x^{\prime}, x^{\prime \prime} ; l_{T}^{2}\right)\left[\left(\frac{1}{2}-\frac{2}{9}\right) \frac{\vec{e}^{( \pm)} \cdot \vec{k}_{T}}{k_{T}^{2}+m_{c}^{2}}-\frac{1}{36} \frac{e^{( \pm)} \cdot\left(\vec{k}_{T}-\vec{l}_{T}\right)}{m_{c}^{2}+\left(\vec{k}_{T}-\vec{l}_{T}\right)^{2}}\right. \\
& \left.-\frac{1+\alpha_{k}}{4} \frac{\vec{e}^{( \pm)} \cdot\left(\vec{k}_{T}-\left(1+\alpha_{k}\right) \vec{l}_{T}\right)}{m_{c}^{2}+\left(\vec{k}_{T}-\left(1+\alpha_{k}\right) \vec{l}_{T}\right)^{2}}+\frac{\alpha_{k}}{4} \frac{e^{( \pm)} \cdot\left(\vec{k}_{T}-\alpha_{k} \vec{l}_{T}\right)}{m_{c}^{2}+\left(\vec{k}_{T}-\alpha_{k} \vec{l}_{T}\right)^{2}}\right]
\end{aligned}
$$




$$
\begin{aligned}
\mathcal{I}_{c}^{\prime}= & \frac{1}{\pi} \int \frac{d^{2} \vec{l}_{T}}{\left(l_{T}^{2}\right)^{2}} f\left(x^{\prime}, x^{\prime \prime} ; l_{T}^{2}\right)\left[\left(\frac{1}{2}-\frac{2}{9}\right) \frac{1}{k_{T}^{2}+m_{c}^{2}}-\frac{1}{36} \frac{1}{m_{c}^{2}+\left(\vec{k}_{T}-\vec{l}_{T}\right)^{2}}\right. \\
& \left.-\frac{1+\alpha_{k}}{4} \frac{1}{m_{c}^{2}+\left(\vec{k}_{T}-\left(1+\alpha_{k}\right) \vec{l}_{T}\right)^{2}}+\frac{\alpha_{k}}{4} \frac{1}{m_{c}^{2}+\left(\vec{k}_{T}-\alpha_{k} \vec{l}_{T}\right)^{2}}\right]
\end{aligned}
$$

If we approximate the differential off-diagonal gluon distribution function $f\left(x^{\prime}, x^{\prime \prime} ; l_{T}^{2}\right)$ by the usual diagonal differential gluon distribution function $f_{g}\left(x ; l_{T}^{2}\right)$, the above integrations will then be reduced to, after integrating over the azimuth angle of $\vec{l}_{T}$,

$$
\mathcal{T}_{c}^{( \pm)}=\pi e^{( \pm)} \cdot \vec{k}_{T} \mathcal{I}_{c}
$$

where

$$
\begin{aligned}
\mathcal{I}_{c}= & \int \frac{d^{2} \vec{l}_{T}}{\left(l_{T}^{2}\right)^{2}} f_{g}\left(x ; l_{T}^{2}\right)\left[\frac{5}{18} \frac{1}{m_{T}^{2}}-\frac{5}{36} \frac{1}{k_{T}^{2}}-\frac{1}{72} \frac{k_{T}^{2}-m_{c}^{2}-l_{T}^{2}}{m_{1}^{2}}\right. \\
& \left.-\frac{1+\alpha_{k}}{8} \frac{k_{T}^{2}-m_{c}^{2}-\left(1+\alpha_{k}\right)^{2} l_{T}^{2}}{m_{2}^{2}}+\frac{\alpha_{k}}{8} \frac{k_{T}^{2}-m_{c}^{2}-\alpha_{k}^{2} l_{T}^{2}}{m_{3}^{2}}\right],
\end{aligned}
$$

where

$$
\begin{aligned}
& m_{T}^{2}=k_{T}^{2}+m_{c}^{2}, \quad m_{1}^{2}=\sqrt{\left(m_{T}^{2}+l_{T}^{2}\right)^{2}-k_{T}^{2} l_{T}^{2}}, \quad m_{2}^{2}=\sqrt{\left(m_{T}^{2}+\left(1+\alpha_{k}\right)^{2} l_{T}^{2}\right)^{2}-\left(1+\alpha_{k}\right)^{2} k_{T}^{2} l_{T}^{2}}, \\
& m_{3}^{2}=\sqrt{\left(m_{T}^{2}+\alpha_{k}^{2} l_{T}^{2}\right)^{2}-\alpha_{k}^{2} k_{T}^{2} l_{T}^{2}},
\end{aligned}
$$

and

$$
\mathcal{I}_{c}^{\prime}=\int \frac{d^{2} \vec{l}_{T}}{\left(l_{T}^{2}\right)^{2}} f_{g}\left(x ; l_{T}^{2}\right)\left[\frac{5}{18} \frac{1}{m_{T}^{2}}-\frac{1}{36} \frac{1}{m_{1}^{2}}-\frac{1+\alpha_{k}}{4} \frac{1}{m_{2}^{2}}+\frac{\alpha_{k}}{4} \frac{1}{m_{3}^{2}}\right] .
$$

So, the amplitude squared for the partonic process $g p \rightarrow c \bar{c} p$ will then be reduced to, after averaging over the spin and color degrees of freedom,

$$
\overline{\mid \mathcal{A}}^{2}=\frac{9}{4} \alpha_{s}^{3}(4 \pi)^{3} \pi^{2} s^{2} \frac{m_{T}^{2}}{M_{X}^{2}}\left[\left(1-\frac{2 k_{T}^{2}}{M_{X}^{2}}\right) k_{T}^{2}\left|\mathcal{I}_{c}\right|^{2}+m_{c}^{2}\left|\mathcal{I}_{c}^{\prime}\right|^{2}\right] .
$$

From the above results, we can see that the integrals of Eqs. (37) and (39) are proportional to $1 / l_{T}^{2}$ in the limit of $l_{T}^{2} \rightarrow 0$. That is to say that there exist large logarithmic contributions from the integration region of $1 / R_{N}^{2} \ll l_{T}^{2} \ll m_{T}^{2}$ for the integration over $l_{T}^{2}$ as in Ref. [13]. So, we can expand the integrals of Eqs. (37) and (39) in terms of $l_{T}^{2}$ to get the leading logarithmic contribution to the amplitude. In the limit of $l_{T}^{2} \rightarrow 0$, the parameters $m_{1}^{2}, m_{2}^{2}$ and $m_{3}^{2}$ scale as,

$$
\begin{aligned}
\frac{1}{m_{1}^{2}} & \approx \frac{1}{m_{T}^{2}}\left[1-\frac{m_{c}^{2}-k_{T}^{2}}{m_{T}^{2}} \frac{l_{T}^{2}}{m_{T}^{2}}\right], \\
\frac{1}{m_{2}^{2}} & \approx \frac{1}{m_{T}^{2}}\left[1-\frac{m_{c}^{2}-k_{T}^{2}}{m_{T}^{2}} \frac{\left(1+\alpha_{k}\right)^{2} l_{T}^{2}}{m_{T}^{2}}\right], \\
\frac{1}{m_{3}^{2}} & \approx \frac{1}{m_{T}^{2}}\left[1-\frac{m_{c}^{2}-k_{T}^{2}}{m_{T}^{2}} \frac{\alpha_{k}^{2} l_{T}^{2}}{m_{T}^{2}}\right] .
\end{aligned}
$$


Under this approximation, the integrations Eqs. (37) and (39) will then be related to the integrated gluon distribution function $x g\left(x ; Q^{2}\right)$,

$$
\begin{aligned}
\mathcal{I}_{c} & \approx \frac{2 m_{c}^{2}}{36\left(m_{T}^{2}\right)^{3} M_{X}^{2}}\left(10 M_{X}^{2}-27 m_{T}^{2}\right) x g\left(x ; m_{T}^{2}\right), \\
\mathcal{I}^{\prime}{ }_{c} & \approx \frac{m_{c}^{2}-k_{T}^{2}}{36\left(m_{T}^{2}\right)^{3} M_{X}^{2}}\left(10 M_{X}^{2}-27 m_{T}^{2}\right) x g\left(x ; m_{T}^{2}\right) .
\end{aligned}
$$

Substituting the above results into Eq. (40), we can then reproduce the leading logarithmic approximation result for the diffractive charm jet production process at hadron colliders which has been calculated in 113 .

\section{C. $q p \rightarrow q g p$ process}

We first give the Sudakov parameters for the loop mentum $l$ for this process (Fig. 3),

$$
\begin{aligned}
\alpha_{l} & =-\frac{l_{T}^{2}}{s}, \\
\beta_{l} & =\frac{2\left(k_{T}, l_{T}\right)-l_{T}^{2}}{\alpha_{k} s}, \quad \text { for Diag.1, } 2,6 \\
& =\frac{2\left(k_{T}, l_{T}\right)+l_{T}^{2}}{\left(1+\alpha_{k}\right) s}, \quad \text { for Diag.5, } 7,8, \\
& =-\frac{M_{X}^{2}-l_{T}^{2}}{s}, \quad \text { for Diag.3, } 4,9 .
\end{aligned}
$$

And the imaginary part of the amplitude $\mathcal{A}(q p \rightarrow q g p)$ for each diagram of Fig. 3 has the following general form,

$$
\operatorname{Im} \mathcal{A}=C_{F}\left(T_{i j}^{a}\right) \int \frac{d^{2} l_{T}}{\left(l_{T}^{2}\right)^{2}} F \times \bar{u}_{i}(u-k) \Gamma_{\mu} u_{j}(q),
$$

where $C_{F}$ is the color factor for each diagram. $a$ is the color index of the incident gluon. $\Gamma_{\mu}$ represents some $\gamma$ matrices including one propagator. $F$ is the same as in Eq. (8).

In [21], we calculated the diffractive photon production process $q p \rightarrow \gamma q p$, in which the Feynman diagrams are similar to the first four diagrams of Fig. 3. In that paper, we calculate the cross section by directly squaring the partonic process amplitude. However, in the calculations here for the partonic process $q p \rightarrow q g p$ because there are additional five diagrams contribution, it is not convenient to directly square the amplitude. Following the above subsections, we calculate the amplitude by employing the helicity amplitude method. Furthermore, we will show that by using the helicity amplitude method we can reproduce the cross section formula for the diffractive photon production process [21].

For the massless quark spinors, we use the definition of Eq. (10). For the polarization vector of the outgoing gluon (its momentum is $k+q$ ), following the method of Ref. [17], we find that it is convenient to choose

$$
\phi^{( \pm)}=N_{e}\left[(\not k+\not q) \not q \not p\left(1 \mp \gamma_{5}\right)+\not p \not q(\not k+\not q)\left(1 \pm \gamma_{5}\right)\right]
$$


The normalization factor $N_{e}$ equals to

$$
N_{e}=\frac{1}{s \sqrt{2 k_{T}^{2}}}
$$

With this definition (46), we can easily get the scalar products between the four-momenta and the polarization vector $e$ as

$$
e \cdot p=0, \quad e \cdot q=N_{e} \frac{k_{T}^{2} s}{1+\alpha_{k}}, \quad e \cdot k_{T}=-N_{e} k_{T}^{2} s, \quad e \cdot l_{T}=-N_{e}\left(k_{T}, l_{T}\right) s .
$$

And then the helicity amplitudes have the following general forms [19,

$$
\bar{u}_{ \pm}\left(p_{f}\right) Q u_{ \pm}\left(p_{i}\right)=\frac{\operatorname{Tr}\left[Q \not p_{i} \not h \not p_{f}\left(1 \mp \gamma_{5}\right)\right]}{4 \sqrt{\left(n \cdot p_{i}\right)\left(n \cdot p_{f}\right)}}
$$

where $n$ is an arbitrary massless 4 -vector, which is also set to be $n=p$. Using this formula (49), we can calculate the helicity amplitude $\mathcal{A}\left(\lambda_{1}, \lambda_{2}, \lambda_{3}\right)$ for the diffractive process $q p \rightarrow$ qgp. Here $\lambda_{1}$ represents the helicity of the incident quark; $\lambda_{2}$ and $\lambda_{3}$ represent the helicities of the outgoing gluon and quark respectively.

For the first four diagrams, to sum up together, the imaginary part of the amplitude $\mathcal{A}(+,+,+)$ is

$$
\operatorname{Im} \mathcal{A}^{1234}(+,+,+)=\alpha_{k}^{2}\left(1+\alpha_{k}\right) \mathcal{N}_{q} \times \int \frac{d^{2} \vec{l}_{T}}{\left(l_{T}^{2}\right)^{2}} f\left(x^{\prime}, x^{\prime \prime} ; l_{T}^{2}\right)\left(\frac{2}{9}-\frac{-1}{36} \frac{k_{T}^{2}-\left(1+\alpha_{k}\right)\left(k_{T}, l_{T}\right)}{\left(\vec{k}_{T}-\left(1+\alpha_{k}\right) \vec{l}_{T}\right)^{2}}\right),
$$

where $\frac{2}{9}$ and $\frac{-1}{36}$ are the color factors for Diags.1,4 and Diags.2,3 respectively, and $\mathcal{N}_{q}$ is defined as

$$
\mathcal{N}_{q}=\frac{3 s}{\sqrt{-2 \alpha_{k} k_{T}^{2}}} g_{s}^{3} T_{i j}^{a}
$$

The other helicity amplitudes for the first four diagrams have the similar forms as (50),

$$
\begin{aligned}
& \operatorname{Im} \mathcal{A}^{1234}(-,-,-)=\operatorname{Im} \mathcal{A}^{1234}(+,+,+), \\
& \operatorname{Im} \mathcal{A}^{1234}(+,-,+)=\operatorname{Im} \mathcal{A}^{1234}(-,+,-)=\frac{-1}{\alpha_{k}} \operatorname{Im} \mathcal{A}^{1234}(+,+,+) .
\end{aligned}
$$

These amplitude expressions Eq. (50) can also serve as the calculations of the amplitude for the diffractive direct photon production process $q p \rightarrow q \gamma p$ [21] except the difference on the color factors. In the direct photon process, the color factors for these four diagrams are the same (they are all $\frac{2}{9}$ ).

\footnotetext{
${ }^{2}$ In Ref. [21], we did not employ the helicity amplitude method. If we use the amplitude expressions Eqs.(50) and (52) (correct the color factors) to calculate the photon production process $q p \rightarrow q \gamma p$, we can get the same result as that in 21]. This can be viewed as a cross check for the methods we used in the calculations.
} 
As discussed in the above subsection of the calculation for the partonic processes $g p \rightarrow$ $q \bar{q} p$, we must first exam the amplitude (45) behavor under the limit of $l_{T}^{2} \rightarrow 0$ in the integration over $l$ to avoid the linear singularities. From Eq. (50), we can see that the amplitude for the diffractive direct photon production process $q p \rightarrow q \gamma p$ is exact zero at $l_{T}^{2} \rightarrow 0$. However, for the process $q p \rightarrow q g p$ the amplitude for the first four diagrams is not exact zero in the limit $l_{T}^{2} \rightarrow 0$ due to the inequality of the color factors between them. That is to say, the sum of the first four diagrams is not free of linear singularities. So, the contributions from the last five diagrams of Fig. 3 must cancel out the linear singularity which rises from the first four diagrams to guarantee the total sum of the amplitude from all of the diagrams free of linear singularities.

Finally, by adding up all of the nine diagrams of Fig.2, the imaginary parts of the amplitudes are

$$
\begin{aligned}
& \operatorname{Im} \mathcal{A}(+,+,+)=\operatorname{Im} \mathcal{A}(-,-,-)=\frac{\alpha_{k}^{2}}{4} \mathcal{N}_{q} \times \mathcal{T} \\
& \operatorname{Im} \mathcal{A}(+,-,+)=\operatorname{Im} \mathcal{A}(-,+,-)=-\frac{\alpha_{k}}{4} \mathcal{N}_{q} \times \mathcal{T}
\end{aligned}
$$

where

$$
\begin{aligned}
\mathcal{T}= & \int \frac{d^{2} \vec{l}_{T}}{\left(l_{T}^{2}\right)^{2}} f\left(x^{\prime}, x^{\prime \prime} ; l_{T}^{2}\right)\left[\frac{\left(1+\alpha_{k}\right)^{2}}{9} \frac{\left(k_{T}, l_{T}\right)-\left(1+\alpha_{k}\right) l_{T}^{2}}{\left(\vec{k}_{T}-\left(1+\alpha_{k}\right) \vec{l}_{T}\right)^{2}}-\left(1+\alpha_{k}\right) \frac{\left(k_{T}, l_{T}\right)+l_{T}^{2}}{\left(\vec{k}_{T}+\vec{l}_{T}\right)^{2}}\right. \\
& \left.-\alpha_{k} \frac{\left(k_{T}, l_{T}\right)-l_{T}^{2}}{\left(\vec{k}_{T}-\vec{l}_{T}\right)^{2}}+\alpha_{k}^{2} \frac{\left(k_{T}, l_{T}\right)-\alpha_{k} l_{T}^{2}}{\left(\vec{k}_{T}-\alpha_{k} \vec{l}_{T}\right)^{2}}\right] .
\end{aligned}
$$

From the above results, we can see that in the integration of the amplitude the linear singularity from different diagrams are canceled out by each other, which will guarantee there is no linear singularity in the total sum.

Following the argument in the above subsection of the calculation for the partonic process $g p \rightarrow q \bar{q} p$, we can also approximate the differential off-diagonal gluon distribution function $f\left(x^{\prime}, x^{\prime \prime} ; l_{T}^{2}\right)$ by the usual diagonal differential gluon distribution function $f_{g}\left(x ; l_{T}^{2}\right)$ in the integration $\mathcal{T}$, by which we can further simplify the integration of $\mathcal{T}$. After integrated over the azimuth angle of $\vec{l}_{T}$, the integration $\mathcal{T}$ will then be

$$
\begin{aligned}
\mathcal{T}= & \pi \int \frac{d l_{T}^{2}}{\left(l_{T}^{2}\right)^{2}} f_{g}\left(x ; l_{T}^{2}\right)\left[\frac{1+\alpha_{k}}{9}\left(\frac{1}{2}-\frac{k_{T}^{2}-\left(1+\alpha_{k}\right) l_{T}^{2}}{2\left|k_{T}^{2}-\left(1+\alpha_{k}\right) l_{T}^{2}\right|}\right)+\left(\frac{1}{2}-\frac{k_{T}^{2}-l_{T}^{2}}{2\left|k_{T}^{2}-l_{T}^{2}\right|}\right)\right. \\
& \left.+\alpha_{k}\left(\frac{1}{2}-\frac{k_{T}^{2}-\alpha_{k} l_{T}^{2}}{2\left|k_{T}^{2}-\alpha_{k} l_{T}^{2}\right|}\right)\right] .
\end{aligned}
$$

In the above integration, if $l_{T}^{2}<k_{T}^{2} /\left(1+\alpha_{k}\right)^{2}$ the first term of the integration over $l_{T}^{2}$ will be zero; if $l_{T}^{2}<k_{T}^{2}$ the second term will be zero; if $l_{T}^{2}<k_{T}^{2} / \alpha_{k}^{2}$ the third term will be zero. So, the dominant regions contributing to the three integration terms are $l_{T}^{2} \sim k_{T}^{2} /\left(1+\alpha_{k}\right)^{2}$, $l_{T}^{2} \sim k_{T}^{2}$, and $l_{T}^{2} \sim k_{T}^{2} / \alpha_{k}^{2}$ respectively. Approximately, by ignoring some evolution effects of the differential gluon distribution function $f_{g}\left(x ; l_{T}^{2}\right)$ in the above dominant integration regions, we get the following results for the integration $\mathcal{T}$,

$$
\mathcal{T}=\frac{\pi}{k_{T}^{2}}\left[f_{g}\left(x ; k_{T}^{2}\right)+\frac{\left(1+\alpha_{k}\right)^{3}}{9} f_{g}\left(x ; \frac{k_{T}^{2}}{\left(1+\alpha_{k}\right)^{2}}\right)+\alpha_{k}^{3} f_{g}\left(x ; \frac{k_{T}^{2}}{\alpha_{k}^{2}}\right)\right] .
$$


Obtained the formula for the integration $\mathcal{T}$, the amplitude squared for the partonic process $q p \rightarrow q g p$ will be reduced to, after averaging over the spin and color degrees of freedom,

$$
\overline{\mid \mathcal{A}}^{2}=\frac{\alpha_{s}^{3}(4 \pi)^{3}}{24} \frac{1+\alpha_{k}^{2}}{M_{X}^{2}\left(1+\alpha_{k}\right)} s^{2}|\mathcal{T}|^{2} .
$$

And the cross section for the partonic process $q p \rightarrow q g p$ is

$$
\begin{aligned}
\left.\frac{d \hat{\sigma}(q p \rightarrow q g p)}{d t}\right|_{t=0}= & \int_{M_{X}^{2}>4 k_{T}^{2}} d M_{X}^{2} d k_{T}^{2} d \alpha_{k}\left[\delta\left(\alpha_{k}-\alpha_{1}\right)+\delta\left(\alpha_{k}-\alpha_{2}\right)\right] \\
& \frac{\alpha_{s}^{3}}{96\left(M_{X}^{2}\right)^{2}} \frac{1+\alpha_{k}^{2}}{1+\alpha_{k}} \frac{1}{\sqrt{1-\frac{4 k_{T}^{2}}{M_{X}^{2}}}}|\mathcal{T}|^{2}
\end{aligned}
$$

where $\alpha_{1,2}$ are the solutions of the following equations,

$$
\alpha(1+\alpha)+\frac{k_{T}^{2}}{M_{X}^{2}}=0
$$

The integral bound $M_{X}^{2}>4 k_{T}^{2}$ in (58) shows that the dominant contribution of the integration over $M_{X}^{2}$ comes from the region of $M_{X}^{2} \sim 4 k_{T}^{2}$. Using Eq. (舟), this indicates that in this dominant region $\alpha_{k}$ is of order of 1 . So, in the integration $\mathcal{T}$ the differential gluon distribution function $f_{g}\left(x ; Q^{2}\right)$ of the three terms can approximately take their values at the same scale of $Q^{2}=k_{T}^{2}$. That is, the integration $\mathcal{T}$ is then simplified to

$$
\mathcal{T}=\frac{\pi}{9 k_{T}^{2}} f_{g}\left(x ; k_{T}^{2}\right)\left(1+\alpha_{k}\right)\left(10-7 \alpha_{k}+10 \alpha_{k}^{2}\right)
$$

\section{D. $g p \rightarrow g g p$ process}

For the partonic process $g p \rightarrow g g p$, there are twelve diagrams in the leading order contributions as shown in Fig. 4. The first nine diagrams are due to the existence of the three-gluon interaction vertex, and the last three diagrams are due to the existence of the four-gluon interaction vertex. But it will be shown in the following calculations, the last three diagrams do not contribute under some choice of the polarizations of the three external gluons.

As usual, we first give the Sudakov parameters for the loop momentum $l$,

$$
\begin{aligned}
\alpha_{l} & =-\frac{l_{T}^{2}}{s}, \\
\beta_{l} & =\frac{2\left(k_{T}, l_{T}\right)-l_{T}^{2}}{\alpha_{k} s}, \quad \text { for Diag.1, 4, 6, 10, } \\
& =\frac{2\left(k_{T}, l_{T}\right)+l_{T}^{2}}{\left(1+\alpha_{k}\right) s}, \quad \text { for Diag.2, 3, 5, 11, } \\
& =-\frac{M_{X}^{2}-l_{T}^{2}}{s}, \quad \text { for Diag.7, 8, 9, } 12 .
\end{aligned}
$$


And the imaginary part of the amplitude $\mathcal{A}(g p \rightarrow g g p)$ for each diagram of Fig. 4 has the following general form,

$$
\operatorname{Im} \mathcal{A}=C_{F} f_{a b c} \int \frac{d^{2} l_{T}}{\left(l_{T}^{2}\right)^{2}} G\left(e_{1}, e_{2}, e_{3}\right) \times F,
$$

where $C_{F}$ is the color factor for each diagram. $a, b, c$ are the color indexes for the incident gluon and the two outgoing gluons respectively, and $f_{a b c}$ are the antisymmetric $S U(3)$ structure constants. $G\left(e_{1}, e_{2}, e_{3}\right)$ represents the interaction part including one propagator for the first nine diagrams, where $e_{1}, e_{2}, e_{3}$ are the polarization vectors for the incident gluon and the two outgoing gluons. $F$ in the integral is the same as that in Eq.(8).

The color factors $C_{F}$ for the twelve diagrams are

$$
\begin{array}{ll}
C_{F}=\frac{1}{2}, & \text { for Diag.1, } 7, \\
C_{F}=-\frac{1}{2}, & \text { for Diag.2, } \\
C_{F}=-\frac{1}{4}, & \text { for Diag.3, 6, 9, } \\
C_{F}=\frac{1}{4}, & \text { for Diag.4, 5, 8, } \\
C_{F}=\frac{3}{4}, & \text { for Diag.10, } 11,12 .
\end{array}
$$

Following the calculation method used in the above subsections, we employ the helicity amplitude method to calculate the amplitude Eq.(62). For the polarization vector of the incident gluon, which is transversely polarized, we use the same definition in Eq. (11). For the two outgoing gluons, we choose their polarization vectors as [17]

$$
\begin{aligned}
& \phi_{2}^{( \pm)}=N_{e}\left[(\not k+\not q) \not q \not p\left(1 \mp \gamma_{5}\right)+\not p \not q(\not k+\not h)\left(1 \pm \gamma_{5}\right)\right], \\
& \phi_{3}^{( \pm)}=N_{e}\left[(\not l-\not k) \not q \not p\left(1 \mp \gamma_{5}\right)+\not p \not q(\not l-\not k)\left(1 \pm \gamma_{5}\right)\right] .
\end{aligned}
$$

The normalization factor $N_{e}$ has the same form as in Eq.(47). Under the above choice of the polarization vectors for the external gluons, we can easily find that they are satisfied the following equations,

$$
p \cdot e_{1}=p \cdot e_{2}=p \cdot e_{3}=0 .
$$

With these relations, we can further find that the last three diagrams do not contribute to the partonic process $g p \rightarrow g g p$.

For the first nine diagrams, there are two helicity amplitudes among the eight helicity amplitudes do not contribute in the context of the above choice of the polarizations of the external gluons, i.e.,

$$
\operatorname{Im} \mathcal{A}(+,+,+)=\operatorname{Im} \mathcal{A}(-,-,-)=0 .
$$

In the expression of the amplitude $\mathcal{A}\left(\lambda\left(e_{1}\right), \lambda\left(e_{2}\right), \lambda\left(e_{3}\right)\right), \lambda$ denote the helicities for the three gluons respectively. The other six helicity amplitudes are divided into the following three different sets, 


$$
\begin{aligned}
& \operatorname{Im} \mathcal{A}(+,-,-) \sim \operatorname{Im} \mathcal{A}(-,+,+), \\
& \operatorname{Im} \mathcal{A}(+,-,+) \sim \operatorname{Im} \mathcal{A}(-,+,-), \\
& \operatorname{Im} \mathcal{A}(+,+,-) \sim \operatorname{Im} \mathcal{A}(-,-,+) .
\end{aligned}
$$

For the first helicity amplitudes set, $\operatorname{Im} \mathcal{A}( \pm, \mp, \mp)$, to sum up all of the nine diagrams, we get

$$
\operatorname{Im} \mathcal{A}( \pm, \mp, \mp)=\mathcal{N}^{\prime} \pi e_{1}^{( \pm)} \cdot k_{T} \mathcal{I}_{g}
$$

where $\mathcal{N}^{\prime}$ is defined as

$$
\mathcal{N}^{\prime}=\frac{3}{4} \frac{s}{k_{T}^{2}} g_{s}^{3} f_{a b c}
$$

And the integration $\mathcal{I}_{g}$ is

$$
\begin{aligned}
\mathcal{I}_{g}= & \frac{1}{\pi} \int \frac{d^{2} \vec{l}_{T}}{\left(l_{T}^{2}\right)^{2}} f\left(x^{\prime}, x^{\prime \prime} ; l_{T}^{2}\right)\left[-\left(1+\alpha_{k}\right) \frac{k_{T}^{2}+\left(k_{T}, l_{T}\right)}{\left(\vec{k}_{T}+\vec{l}_{T}\right)^{2}}+\alpha_{k} \frac{k_{T}^{2}-\left(k_{T}, l_{T}\right)}{\left(\vec{k}_{T}-\vec{l}_{T}\right)^{2}}\right. \\
& \left.+\left(1+\alpha_{k}\right)^{2} \frac{k_{T}^{2}-\left(1+\alpha_{k}\right)\left(k_{T}, l_{T}\right)}{\left(\vec{k}_{T}-\left(1+\alpha_{k}\right) \vec{l}_{T}\right)^{2}}-\alpha_{k}^{2} \frac{k_{T}^{2}-\alpha_{k}\left(k_{T}, l_{T}\right)}{\left(\vec{k}_{T}-\alpha_{k} \vec{l}_{T}\right)^{2}}\right] \\
= & \frac{1}{\pi} \int \frac{d^{2} \vec{l}_{T}}{\left(l_{T}^{2}\right)^{2}} f\left(x^{\prime}, x^{\prime \prime} ; l_{T}^{2}\right)\left[\left(1+\alpha_{k}\right) \frac{\left(k_{T}, l_{T}\right)+l_{T}^{2}}{\left(\vec{k}_{T}+\vec{l}_{T}\right)^{2}}+\alpha_{k} \frac{\left(k_{T}, l_{T}\right)-l_{T}^{2}}{\left(\vec{k}_{T}-\vec{l}_{T}\right)^{2}}\right. \\
& \left.-\left(1+\alpha_{k}\right)^{2} \frac{\left(k_{T}, l_{T}\right)-\left(1+\alpha_{k}\right) l_{T}^{2}}{\left(\vec{k}_{T}-\left(1+\alpha_{k}\right) \vec{l}_{T}\right)^{2}}+\alpha_{k}^{2} \frac{\left(k_{T}, l_{T}\right)-\alpha_{k} l_{T}^{2}}{\left(\vec{k}_{T}-\alpha_{k} \vec{l}_{T}\right)^{2}}\right] .
\end{aligned}
$$

From the above equations, we can check that there is no linear singularity at the limit of $l_{T}^{2} \rightarrow 0$ in the integration of the amplitude over the loop momentum. The first term of the integration $\mathcal{I}_{g}$ in Eq. (70) comes from the contribution of Diag.3; the second term comes from Diag.4; the third term comes from Diag.6 and Diag.9; the last term comes from Diag.5 and Diag.8. The contributions from Diags.1, 2 and 7 are canceled out by each other. From (70), we can see that the linear singularities coming from the four terms are canceled out by each other. The final result for the amplitude is now free of linear singularity. We must emphasize here that only the total sum of the contributions from all of the diagrams is free of linear singularity. The separation of these diagrams will cause linear singularity.

Using the same approximations used in the above subsections, i.e., approximating the differential off-diagonal gluon distribution function $f\left(x^{\prime}, x^{\prime \prime} ; l_{T}^{2}\right)$ by the usual diagonal differential gluon distribution function $f_{g}\left(x ; l_{T}^{2}\right)$, we can further simplify the integration of $\mathcal{I}_{g}$. After integrated over the azimuth angle of $\vec{l}_{T}$, this integration will then be

$$
\begin{aligned}
\mathcal{I}_{g}= & \int \frac{d l_{T}^{2}}{\left(l_{T}^{2}\right)^{2}} f_{g}\left(x ; l_{T}^{2}\right)\left[\left(\frac{1}{2}-\frac{k_{T}^{2}-l_{T}^{2}}{2\left|k_{T}^{2}-l_{T}^{2}\right|}\right)-\left(1+\alpha_{k}\right)\left(\frac{1}{2}-\frac{k_{T}^{2}-\left(1+\alpha_{k}\right) l_{T}^{2}}{2\left|k_{T}^{2}-\left(1+\alpha_{k}\right) l_{T}^{2}\right|}\right)\right. \\
& \left.+\alpha_{k}\left(\frac{1}{2}-\frac{k_{T}^{2}-\alpha_{k} l_{T}^{2}}{2\left|k_{T}^{2}-\alpha_{k} l_{T}^{2}\right|}\right)\right] .
\end{aligned}
$$

The above equation shows that the integration $\mathcal{I}_{g}$ here has the similar behavior as that of the integration $\mathcal{T}$ of Eq. (55) in the last subsection. So, the three terms of the above integration 
$\mathcal{I}_{g}$ are dominantly contributed from the integral regions of $l_{T}^{2}$ as $l_{T}^{2} \sim k_{T}^{2} /\left(1+\alpha_{k}\right)^{2}, l_{T}^{2} \sim k_{T}^{2}$, and $l_{T}^{2} \sim k_{T}^{2} / \alpha_{k}^{2}$ respectively. Approximately, we may also ignore the evolution effects of the differential gluon distribution function $f_{g}\left(x ; l_{T}^{2}\right)$ in the above dominant integration regions, and so the integration $\mathcal{I}_{g}$ is reduced to

$$
\mathcal{I}_{g}=\frac{1}{k_{T}^{2}}\left[f_{g}\left(x ; k_{T}^{2}\right)-\left(1+\alpha_{k}\right)^{3} f_{g}\left(x ; \frac{k_{T}^{2}}{\left(1+\alpha_{k}\right)^{2}}\right)+\alpha_{k}^{3} f_{g}\left(x ; \frac{k_{T}^{2}}{\alpha_{k}^{2}}\right)\right] .
$$

For the second helicity amplitudes set, $\operatorname{Im} \mathcal{A}( \pm, \mp, \pm)$, the calculations are more complicated, and the contribution from Diag.3 is

$$
\begin{aligned}
\operatorname{Im} \mathcal{A}^{3}( \pm, \mp, \pm)= & -\mathcal{N}^{\prime}\left(1+\alpha_{k}\right)^{2} \int \frac{d^{2} \vec{l}_{T}}{\left(l_{T}^{2}\right)^{2}} f\left(x^{\prime}, x^{\prime \prime} ; l_{T}^{2}\right) \\
& \frac{\alpha_{k} k_{T}^{2} \vec{e}_{1}^{( \pm)} \cdot\left(\vec{k}_{T}+\vec{l}_{T}\right)+\left(k_{T}^{2}+\left(k_{T}, l_{T}\right)\right) \vec{e}_{1}^{( \pm)} \cdot \vec{k}_{T}}{\left(\vec{k}_{T}+\vec{l}_{T}\right)^{2}}
\end{aligned}
$$

The contribution from Diag.4 is

$$
\begin{aligned}
\operatorname{Im} \mathcal{A}^{4}( \pm, \mp, \pm)= & \mathcal{N}^{\prime} \alpha_{k}\left(1+\alpha_{k}\right) \int \frac{d^{2} \vec{l}_{T}}{\left(l_{T}^{2}\right)^{2}} f\left(x^{\prime}, x^{\prime \prime} ; l_{T}^{2}\right) \\
& \frac{\alpha_{k} k_{T}^{2} \vec{e}_{1}^{( \pm)} \cdot\left(\vec{k}_{T}-\vec{l}_{T}\right)+\left(k_{T}^{2}-\left(k_{T}, l_{T}\right)\right) \vec{e}_{1}^{( \pm)} \cdot \vec{k}_{T}}{\left(\vec{k}_{T}-\vec{l}_{T}\right)^{2}}
\end{aligned}
$$

The contributions from Diag.5 and Diag.8, to sum up together, are

$$
\begin{aligned}
\operatorname{Im} \mathcal{A}^{58}( \pm, \mp, \pm)= & -\mathcal{N}^{\prime} \alpha_{k}\left(1+\alpha_{k}\right) \int \frac{d^{2} \vec{l}_{T}}{\left(l_{T}^{2}\right)^{2}} f\left(x^{\prime}, x^{\prime \prime} ; l_{T}^{2}\right) \\
& \frac{\alpha_{k} k_{T}^{2} \vec{e}_{1}^{( \pm)} \cdot\left(\vec{k}_{T}-\alpha_{k} \vec{l}_{T}\right)+\left(k_{T}^{2}-\alpha_{k}\left(k_{T}, l_{T}\right)\right) \vec{e}_{1}^{( \pm)} \cdot \vec{k}_{T}}{\left(\vec{k}_{T}-\alpha_{k} \vec{l}_{T}\right)^{2}}
\end{aligned}
$$

The contributions from Diag.6 and Diag.9, to sum up together, are

$$
\begin{aligned}
\operatorname{Im} \mathcal{A}^{69}( \pm, \mp, \pm)= & \mathcal{N}^{\prime}\left(1+\alpha_{k}\right)^{2} \int \frac{d^{2} \vec{l}_{T}}{\left(l_{T}^{2}\right)^{2}} f\left(x^{\prime}, x^{\prime \prime} ; l_{T}^{2}\right) \\
& \frac{\alpha_{k} k_{T}^{2} \vec{e}_{1}^{( \pm)} \cdot\left(\vec{k}_{T}-\left(1+\alpha_{k}\right) \vec{l}_{T}\right)+\left(k_{T}^{2}-\left(1+\alpha_{k}\right)\left(k_{T}, l_{T}\right)\right) \vec{e}_{1}^{( \pm)} \cdot \vec{k}_{T}}{\left(\vec{k}_{T}-\left(1+\alpha_{k}\right) \vec{l}_{T}\right)^{2}}
\end{aligned}
$$

The contributions from other three diagrams (Diag.1, Diag.2 and Diag.7) are canceled out by each other. From the above results Eqs.(74.77), we can see that every term has linear singularity at the limit of $l_{T}^{2} \rightarrow 0$ in the integration of the amplitude over $l_{T}^{2}$, while their total sum is free of the linear singularity.

Following the procedure as we do for the helicity amplitude $\mathcal{A}( \pm, \mp, \mp)$ in the above, we can approximate the off-diagonal gluon distribution function $f\left(x^{\prime}, x^{\prime \prime} ; l_{T}^{2}\right)$ by the usual diagonal differential gluon distribution function $f_{g}\left(x ; l_{T}^{2}\right)$. After integrating over the azimuth angle of $\vec{l}_{T}$, to sum up all of Eqs.(74-77), we get the helicity amplitude, 


$$
\operatorname{Im} \mathcal{A}( \pm, \mp, \pm)=\mathcal{N}^{\prime} \pi\left(1+\alpha_{k}\right)^{2} \vec{e}_{1}^{( \pm)} \cdot k_{T} \mathcal{I}_{g},
$$

where $\mathcal{I}_{g}$ is the same as Eq.(72) and then Eq.(73) under the same approximation.

For the third helicity amplitudes set, $\operatorname{Im} \mathcal{A}( \pm, \pm, \mp)$, the calculations are similar to the calculations of $\operatorname{Im} \mathcal{A}( \pm, \mp, \pm)$. The contribution from Diag.3 is

$$
\begin{aligned}
\operatorname{Im} \mathcal{A}^{3}( \pm, \pm, \mp)= & -\mathcal{N}^{\prime} \alpha_{k}\left(1+\alpha_{k}\right) \int \frac{d^{2} \vec{l}_{T}}{\left(l_{T}^{2}\right)^{2}} f\left(x^{\prime}, x^{\prime \prime} ; l_{T}^{2}\right) \\
& \frac{\left(1+\alpha_{k}\right) k_{T}^{2} \vec{e}_{1}^{( \pm)} \cdot\left(\vec{k}_{T}+\vec{l}_{T}\right)-\left(k_{T}^{2}+\left(k_{T}, l_{T}\right)\right) \vec{e}_{1}^{( \pm)} \cdot \vec{k}_{T}}{\left(\vec{k}_{T}+\vec{l}_{T}\right)^{2}} .
\end{aligned}
$$

The contribution from Diag.4 is

$$
\begin{aligned}
\operatorname{Im} \mathcal{A}^{4}( \pm, \pm, \mp)= & \mathcal{N}^{\prime} \alpha_{k}^{2} \int \frac{d^{2} \vec{l}_{T}}{\left(l_{T}^{2}\right)^{2}} f\left(x^{\prime}, x^{\prime \prime} ; l_{T}^{2}\right) \\
& \frac{\left(1+\alpha_{k}\right) k_{T}^{2} \vec{e}_{1}^{( \pm)} \cdot\left(\vec{k}_{T}-\vec{l}_{T}\right)-\left(k_{T}^{2}-\left(k_{T}, l_{T}\right)\right) e_{1}^{( \pm)} \cdot \vec{k}_{T}}{\left(\vec{k}_{T}-\vec{l}_{T}\right)^{2}} .
\end{aligned}
$$

The contributions from Diag.5 and Diag.8, to sum up together, are

$$
\begin{aligned}
\operatorname{Im} \mathcal{A}^{58}( \pm, \pm, \mp)= & -\mathcal{N}^{\prime} \alpha_{k}^{2} \int \frac{d^{2} \vec{l}_{T}}{\left(l_{T}^{2}\right)^{2}} f\left(x^{\prime}, x^{\prime \prime} ; l_{T}^{2}\right) \\
& \frac{\left(1+\alpha_{k}\right) k_{T}^{2} e_{1}^{( \pm)} \cdot\left(\vec{k}_{T}-\alpha_{k} \vec{l}_{T}\right)-\left(k_{T}^{2}-\alpha_{k}\left(k_{T}, l_{T}\right)\right) \vec{e}_{1}^{( \pm)} \cdot \vec{k}_{T}}{\left(\vec{k}_{T}-\alpha_{k} \vec{l}_{T}\right)^{2}} .
\end{aligned}
$$

The contributions from Diag.6 and Diag.9, to sum up together, are

$$
\begin{aligned}
\operatorname{Im} \mathcal{A}^{69}( \pm, \pm, \mp)= & \mathcal{N}^{\prime} \alpha_{k}\left(1+\alpha_{k}\right) \int \frac{d^{2} \vec{l}_{T}}{\left(l_{T}^{2}\right)^{2}} f\left(x^{\prime}, x^{\prime \prime} ; l_{T}^{2}\right) \\
& \frac{\left(1+\alpha_{k}\right) k_{T}^{2} e_{1}^{( \pm)} \cdot\left(\vec{k}_{T}-\left(1+\alpha_{k}\right) \vec{l}_{T}\right)-\left(k_{T}^{2}-\left(1+\alpha_{k}\right)\left(k_{T}, l_{T}\right)\right) \hat{e}_{1}^{( \pm)} \cdot \vec{k}_{T}}{\left(\vec{k}_{T}-\left(1+\alpha_{k}\right) \vec{l}_{T}\right)^{2}} .
\end{aligned}
$$

And also, we find that the contributions from other three diagrams (Diag.1, Diag.2 and Diag.7) are canceled out by each other, and the total sum of Eqs.(79-82) is free of the linear singularity. If we approximate the off-diagonal gluon distribution function $f\left(x^{\prime}, x^{\prime \prime} ; l_{T}^{2}\right)$ by the usual diagonal differential gluon distribution function $f_{g}\left(x ; l_{T}^{2}\right)$, and integrate over the azimuth angle of $\vec{l}_{T}$, their sum will lead to a similar result as in Eq.(78),

$$
\operatorname{Im} \mathcal{A}( \pm, \pm, \mp)=\mathcal{N}^{\prime} \pi \alpha_{k}^{2} \vec{e}_{1}^{( \pm)} \cdot k_{T} \mathcal{I}_{g} .
$$

By summing up all of the helicity amplitudes Eqs.(68), (78) and 83), we will get the amplitude squared for the partonic process $g p \rightarrow g g p$, after averaging over the spin and color degrees of freedom,

$$
\overline{\mid \mathcal{A}}^{2}=\frac{27 \pi^{2} \alpha_{s}^{3}(4 \pi)^{3}}{16} \frac{s^{2}}{k_{T}^{2}}\left(1-\frac{k_{T}^{2}}{M_{X}^{2}}\right)^{2}\left|\mathcal{I}_{g}\right|^{2} .
$$


And the cross section for the partonic process $g p \rightarrow g g p$ is

$$
\left.\frac{d \hat{\sigma}(g p \rightarrow g g p)}{d t}\right|_{t=0}=\int_{M_{X}^{2}>4 k_{T}^{2}} d M_{X}^{2} d k_{T}^{2} \frac{27 \alpha_{s}^{3} \pi^{2}}{32 M_{X}^{2} k_{T}^{2}}\left(1-\frac{k_{T}^{2}}{M_{X}^{2}}\right)^{2}\left|\mathcal{I}_{g}\right|^{2} \frac{1}{\sqrt{1-\frac{4 k_{T}^{2}}{M_{X}^{2}}}}
$$

Following the same argument in the last subsection for the calculations of the partonic process $q p \rightarrow q g p$, we see that the dominant contribution of the integration over $M_{X}^{2}$ comes from the region of $M_{X}^{2} \sim 4 k_{T}^{2}$, where the differential gluon distribution function $f_{g}\left(x ; Q^{2}\right)$ of the three terms in the integration $\mathcal{I}_{g}$ can approximately take their values at the same scale of $Q^{2}=k_{T}^{2}$. That is, the integration $\mathcal{I}_{g}$ is then simplified to

$$
\mathcal{I}_{g}=\frac{1}{k_{T}^{2}}\left(-3 \alpha_{k}\left(1+\alpha_{k}\right)\right) f_{g}\left(x ; k_{T}^{2}\right)=\frac{1}{k_{T}^{2}} \frac{3 k_{T}^{2}}{M_{X}^{2}} f_{g}\left(x ; k_{T}^{2}\right)=\frac{3}{M_{X}^{2}} f_{g}\left(x ; k_{T}^{2}\right) .
$$

\section{NUMERICAL RESULTS}

In this section, we give some numerical results for the diffractive light quark jet and gluon jet production at the Fermilab Tevatron. We will study the $p_{T}$ distribution and $x_{1}$ distribution of the cross section. A more thorough phenomenological study, including a comparison to currently available data at Tevatron on the diffractive dijet production rate, will be presented elsewhere. Again, we emphasize that our numerical results are just for the contribution from the "Pomeron Fragmentation" region $\left(M_{X}^{2} \sim 4 k_{T}^{2}\right)$.

Another important effect which will affect the numerical results is the nonfactorization effect caused by the spectator interactions in the hard diffractive processes in hadron collisions mentioned in the section of Introduction. Here, we use a suppression factor $\mathcal{F}_{S}$ to describe this nonfactorization effect in the hard diffractive processes at hadron colliders [8]. At the Tevatron, the value of $\mathcal{F}_{S}$ may be as small as $\mathcal{F}_{S} \approx 0.1$ [8.9]. That is to say, the total cross section of the diffractive processes at the Tevatron may be reduced down by an order of magnitude due to this nonfactorization effect. In the following numerical calculations, we adopt this suppression factor value to evaluate the diffractive production rate of light quark jet at the Fermilab Tevatron.

The cross section formulas for the three partonic processes have been calculated in the above section. We will use Eqs. (24), (58) and (85) to evaluate the production rates for these processes. In our calculations, the scales for the parton distribution functions and the running coupling constant are both set to be $Q^{2}=k_{T}^{2}$. For the parton distribution functions, we choose the GRV NLO set [22].

The numerical results of the diffractive light quark jet production at the Fermilab Tevatron are plotted in Fig. 5 and Fig. 6. In Fig. 5, we plot the differential cross section $d \sigma /\left.d t\right|_{t=0}$ as a function of the lower bound of the transverse momentum of the light quark jet, $k_{T \min }$. This figure shows that the cross section is sensitive to the transverse momentum cut $k_{T \min }$. The differential cross section decreases over four orders of magnitude as $k_{T \text { min }}$ increases from $5 \mathrm{GeV}$ to $15 \mathrm{GeV}$.

It is interesting to compare the cross section of the diffractive light quark jet production with that of the diffractive charm quark jet production [13], which is also shown in Fig. 5. 
The two curves in this figure show that the production rates of the light quark jet and the charm quark jet in the diffractive processes are in the same order of magnitude. However, we know that the cross section of diffractive heavy quark jet production is related to the integrated gluon distribution function, while the cross section of the light quark jet production is related to the differential gluon distribution function.

In Fig. 6, we plot the differential cross section $d \sigma /\left.d t\right|_{t=0}$ as a function of the lower bound of the momentum fraction of the proton carried by the incident gluon $x_{1 \min }$, where we set $k_{T \min }=5 \mathrm{GeV}$. This figure shows that the dominant contribution comes from the region of $x_{1} \sim 10^{-2}-10^{-1}$. This property is the same as that of the diffractive charm jet production at the Tevatron [13].

In Fig. 7 , we plot the differential cross section $d \sigma /\left.d t\right|_{t=0}$ as a function of the lower bound of the transverse momentum of the gluon jet, $k_{T \min }$. This figure shows that the cross section is sensitive to the transverse momentum cut $k_{T \min }$. We plot separately the contributions from the two subprocesses, $q p \rightarrow q g p$ and $g p \rightarrow g g p$. By comparison, we also plot the cross section of the diffractive light quark jet. The three curves in this figure show that the contribution from the subprocess $g p \rightarrow g g p$ is two orders of magnitude larger than that from the subprocess $q p \rightarrow q g p$ for the diffractive gluon jet production, and the light quark jet production rate is in the same order with that of the subprocess $q p \rightarrow q g p$. This indicates that the diffractive dijet production at hadron colliders dominantly comes from the subprocess $g p \rightarrow g g p$ in the two-gluon exchange model.

In Fig. 8, we plot the differential cross section $d \sigma /\left.d t\right|_{t=0}$ as a function of the lower bound of the momentum fraction of the proton carried by the incident gluon $x_{1 \min }$, where we set $k_{T \min }=5 \mathrm{GeV}$. Fig.5(a) is for the contribution from the subprocess $q p \rightarrow q g p$, and Fig.5(b) is from the subprocess $g p \rightarrow g g p$. These two figures show that the dominant contribution comes from the region of $x_{1} \sim 10^{-2}-10^{-1}$ for the subprocess $g p \rightarrow g g p$, and $x_{1}>10^{-1}$ for the subprocess $q p \rightarrow q g p$. These properties are similar to those of the diffractive charm jet and $W$ boson productions calculated in [13], [14].

\section{CONCLUSIONS}

In this paper, we have calculated the diffractive light quark jet and gluon jet productions at hadron colliders in perturbative QCD by using the two-gluon exchange model. We find that the production cross section is related to the squared differential gluon distribution function $\partial G\left(x ; Q^{2}\right) / \partial \ln Q^{2}$ at the scale of $Q^{2} \sim k_{T}^{2}$, where $k_{T}$ is the transverse momentum of the final state quark or gluon jet. We have also estimated the production rates of the light quark jet and gluon jet in the diffractive processes at the Fermilab Tevatron.

As we know, the large transverse momentum dijet production in the diffractive processes at hadron colliders is important to study the diffractive mechanism and the nature of the Pomeron. The CDF collaboration at the Fermilab Tevatron have reported some results on this process [9]. Up to now, we have calculated all of the dijet production subprocesses in the diffractive processes at hadron colliders, including $g p \rightarrow q \bar{q} p, q p \rightarrow q g p$ and $g p \rightarrow g g p$ processes. In a forthcoming paper, we will compare the available data on the diffractive dijet production cross sections at the Tevatron [9] with the predictions of our model to test the validity of perturbative QCD description of the diffractive processes at hadron colliders. 


\section{ACKNOWLEDGMENTS}

This work was supported in part by the National Natural Science Foundation of China, the Education Commission Ministry of China, and the State Commission of Science and Technology of China. 


\section{REFERENCES}

[1] P.D.B. Collins, An introduction to Regge theory and high energy physics, Cambridge University Press, Cambridge (1977).

[2] M.G. Ryskin, Z. Phys. C37, 89 (1993); M.G. Ryskin, et al., Z. Phys. C76, 231 (1996).

[3] S.J. Brodsky et al., Phys. Rev. D50, 3134 (1994); L. Frankfurt et al., Phys. Rev. D57, 512 (1998).

[4] T. Gehrmann and W.J. Stitling, Z. Phys. C70, 69 (1996); M. Genovese et al., Phys. Lett. B378, 347 (1996); E.M. Levin et al., hep-ph/9606443.

[5] M. Derrick et al, ZEUS collaboration, Phys. Lett. B350 120 (1995); S. Aid et. al., H1 Collaboration, Nucl. Phys. B 472, 3 (1996).

[6] P.V. Landshoff and J.C. Polkinghorne, Nucl. Phys. B33, 221 (1971), B36, 642 (1972); F. Henyey and R. Savit, Phys. Lett. B52, 71 (1974); J.L. Cardy and G.A. Winbow, Phys. Lett. B52, 95 (1974); C. DeTar, S.D. Ellis and P.V. Landshoff, Nucl. Phys. B87, 176 (1975).

[7] J.C. Collins, L. Frankfurt and M. Strikman, Phys. Lett. B307, 161 (1993);

[8] D.E. Soper, talk at DIS97 conference, Chicago, April 1997, hep-ph/9707384.

[9] F. Abe et al., Phys. Rev. Lett. 78, 2698 (1997); F. Abe et al., Phys. Rev. Lett. 79, 2636 (1997).

[10] J.D.Bjorken, Phys. Rev. D45 4077 (1992); D47 10 (1993); E.Gotsman,E. Levin and U. Maor, Phys. Lett. B309 199 (1993); Nucl. Phys. B493 354 (1997); hep-ph/9804404; R.S.Fletcher, Phys. Rev. D48 5162 (1993). A.D. Martin, M.G. Ryskin and V.A. Khoze, Phys. Rev. D56 5867 (1997).

[11] X. Ji, Phys. Rev. Lett. 78, 610 (1997), Phys. Rev. D55, 7114 (1997); A.V. Radyushkin, Phys. Lett. B385, 333 (1996), Phys. Lett. B380, 417 (1996), Phys. Rev. D56, 5524 (1997).

[12] F. Yuan, J.S. Xu, H.A. Peng, and K.T. Chao, Phys. Rev. D58, 114016 (1998).

[13] F. Yuan and K.T. Chao, hep-ph/9810340, to appear in Phys. Rev. D.

[14] F. Yuan and K.T. Chao, hep-ph/9811285.

[15] N.N. Nikolaev and B.G. Zakharov, Phys. Lett. B332, 177 (1994); K. Golec-Biernat, J. Kwiecinski, and A.D. Martin, Preprint DTP/98/12.

[16] G. Alves, E. Levin, and A. Santoro, Phys. Rev. D55, 2683 (1997).

[17] P.De Causmaecker, R.Gastmans, W.Troost, T.T.Wu, Phys. Lett. B105, 215 (1981), Nucl. Phys. B206, 53 (1982); R.Gastmans, W.Troost, T.T.Wu, Nucl. Phys. B291, 731 (1987).

[18] F.A. Berends, P.H. Daverveldt, and R. Kleiss, Nucl. Phys. B253, 441 (1985).

[19] A. L. Bondarev, hep-ph/9710398, and references therein.

[20] P. Hoodbhoy, Phys. Rev. D56, 388 (1997); L. Frankfurt et al., Phys. Lett. B418, 345 (1998); A.D. Martin and M.G. Ryskin, Phys. Rev. D57, 6692 (1998).

[21] F. Yuan, K.T. Chao, hep-ph/9903238.

[22] M. Glück et al. Z. Phys. C67, 433 (1995). 


\section{Figure Captions}

Fig.1. Sketch diagram for the diffractive dijet production at hadron colliders in perturbative QCD. The final state jet lines represent the outgoing quark or gluon lines, and the incident parton from the upper proton (labeled by $x_{1} p_{1}$ ) can be quark or gluon correspondingly.

Fig.2. The lowest order perturbative QCD diagrams for partonic process $g p \rightarrow q \bar{q} p$.

Fig.3. The lowest order perturbative QCD diagrams for partonic process $q p \rightarrow q g p$.

Fig.4. The lowest order perturbative QCD diagrams for partonic process $g p \rightarrow g g p$.

Fig.5. The differential cross section $d \sigma /\left.d t\right|_{t=0}$ for the light quark jet production in the diffractive processes as a function of $k_{T \text { min }}$ at the Fermilab Tevatron, where $k_{T \text { min }}$ is the lower bound of the transverse momentum of the out going quark jet. For the charm quark jet production cross section formula, we take from Ref. [13] and set $m_{c}=1.5 \mathrm{GeV}$.

Fig.6. The differential cross section $d \sigma /\left.d t\right|_{t=0}$ for the light quark jet production as a function of $x_{1 \text { min }}$, where $x_{1 \text { min }}$ is the lower bound of $x_{1}$ in the integration of the cross section.

Fig.7. The differential cross section $d \sigma /\left.d t\right|_{t=0}$ for the gluon jet production in the diffractive processes as a function of $k_{T \text { min }}$.

Fig.8. The differential cross section $d \sigma /\left.d t\right|_{t=0}$ for the gluon jet production as a function of $x_{1 \mathrm{~min}}$, where $x_{1 \mathrm{~min}}$ is the lower bound of $x_{1}$ in the integration of the cross section. (a) is for the contribution from the subprocess $q p \rightarrow q g p$, and (b) is from the subprocess $g p \rightarrow g g p$. 


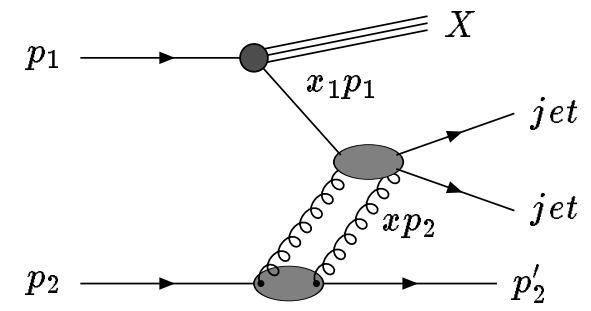

Fig.1 


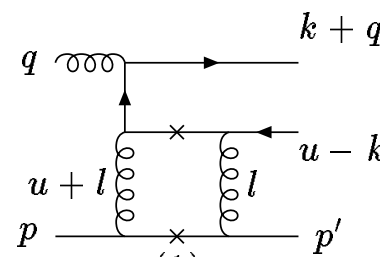

(1)

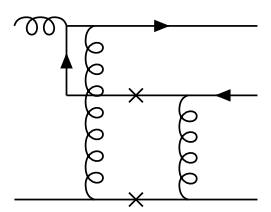

(3)

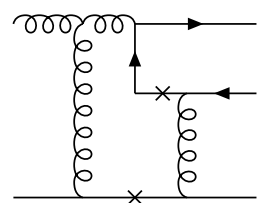

(5)

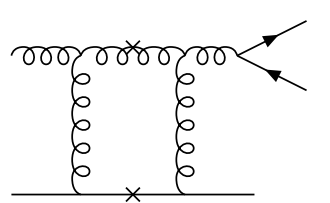

(7)

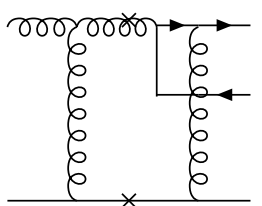

(9)

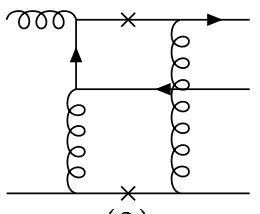

(2)

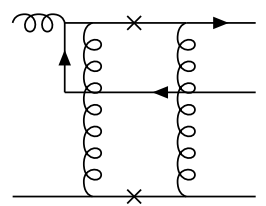

(4)

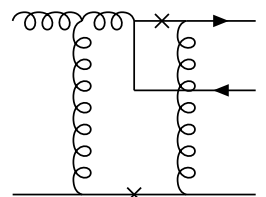

(6)

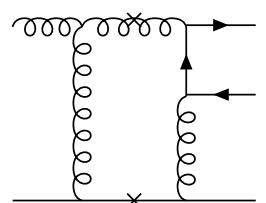

(8)

Fig.2 


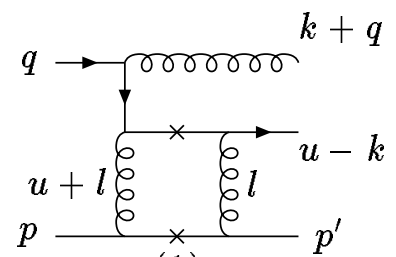

(1)

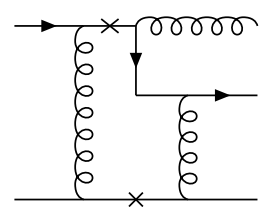

(3)

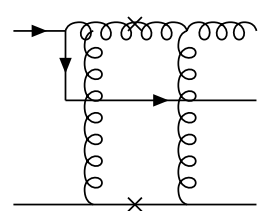

(5)

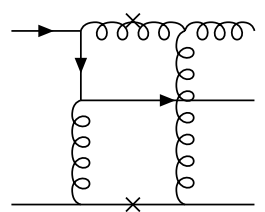

(7)

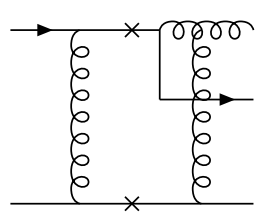

(9)

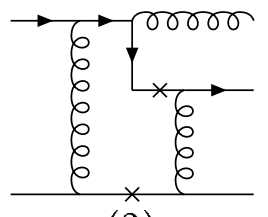

(2)

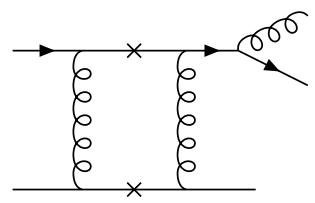

(4)

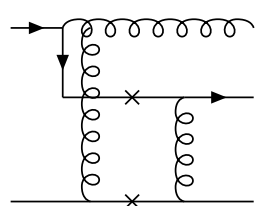

(6)

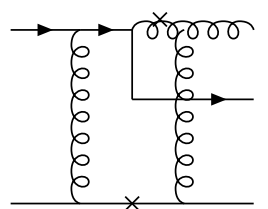

(8)

Fig.3 


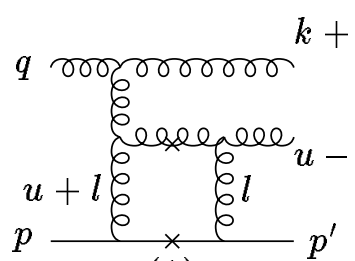

(1)

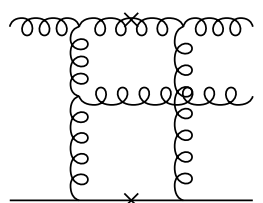

(3)

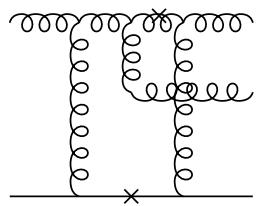

(5)

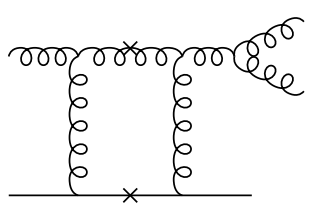

(7)

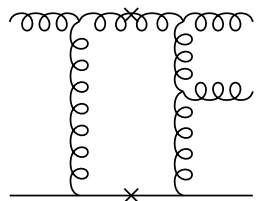

(9)

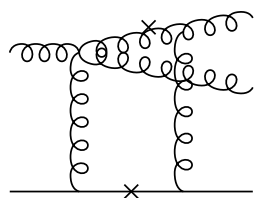

(11)

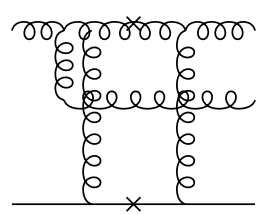

(2)

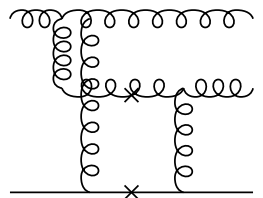

(4)

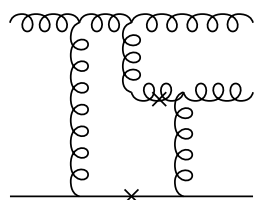

(6)

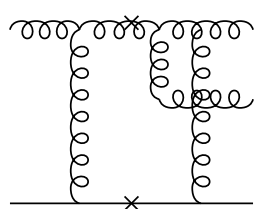

(8)

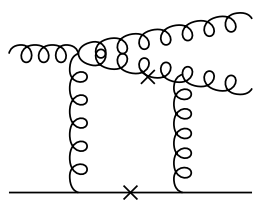

(10)

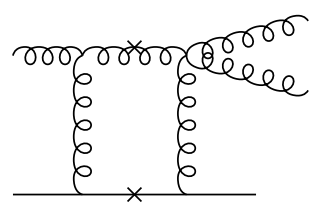

(12)

Fig.4 


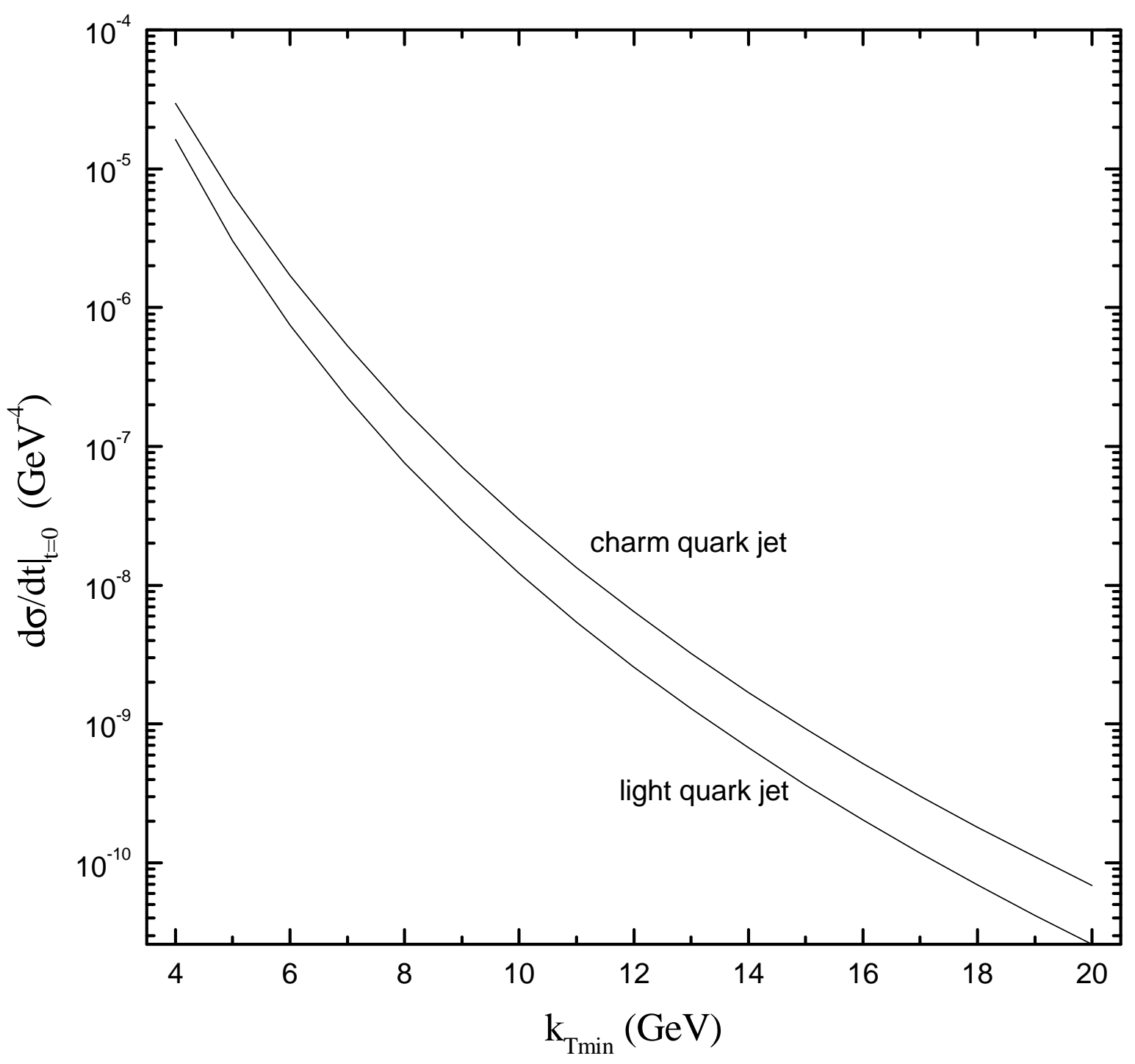

Fig.5 


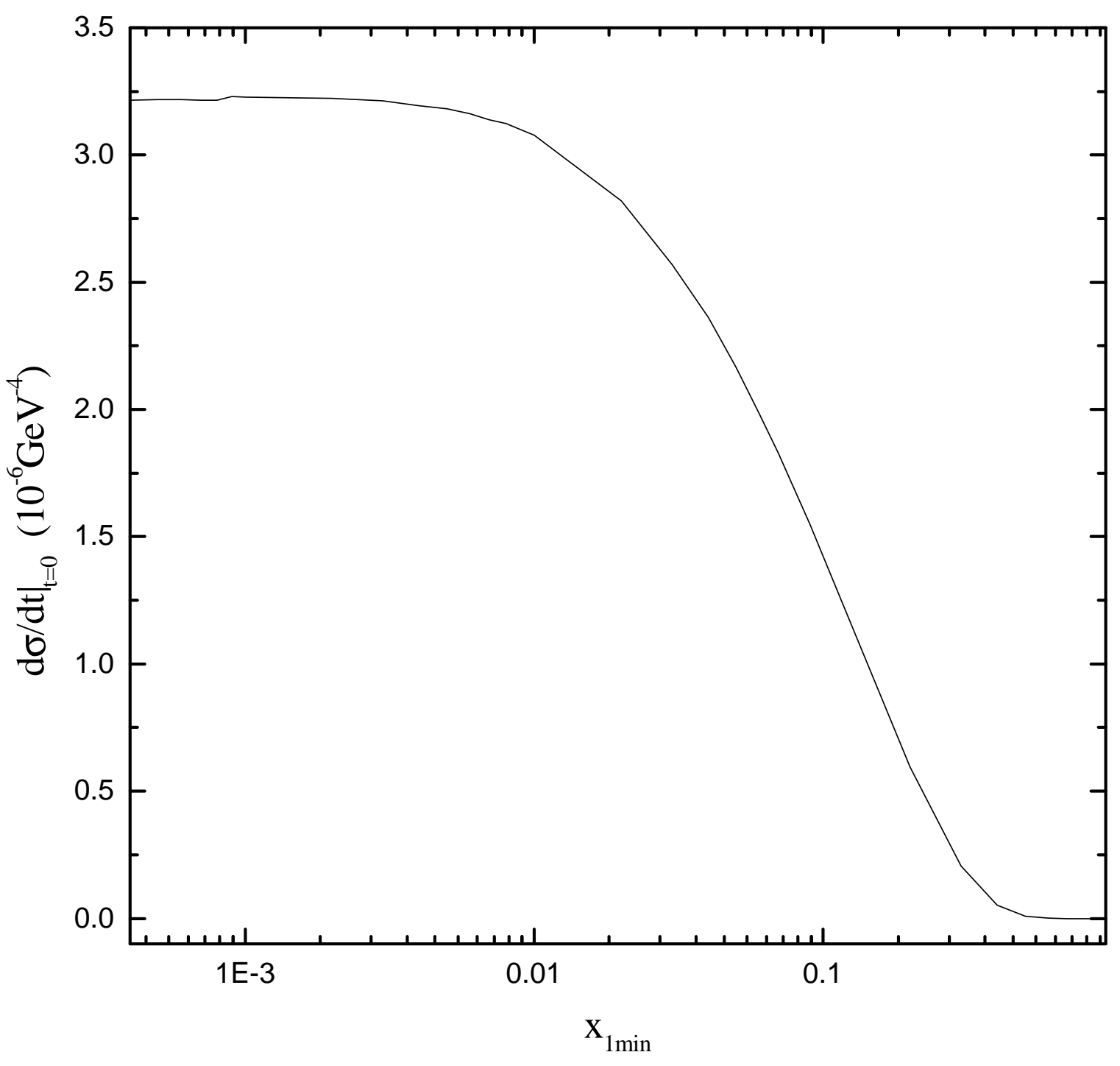

Fig.6 


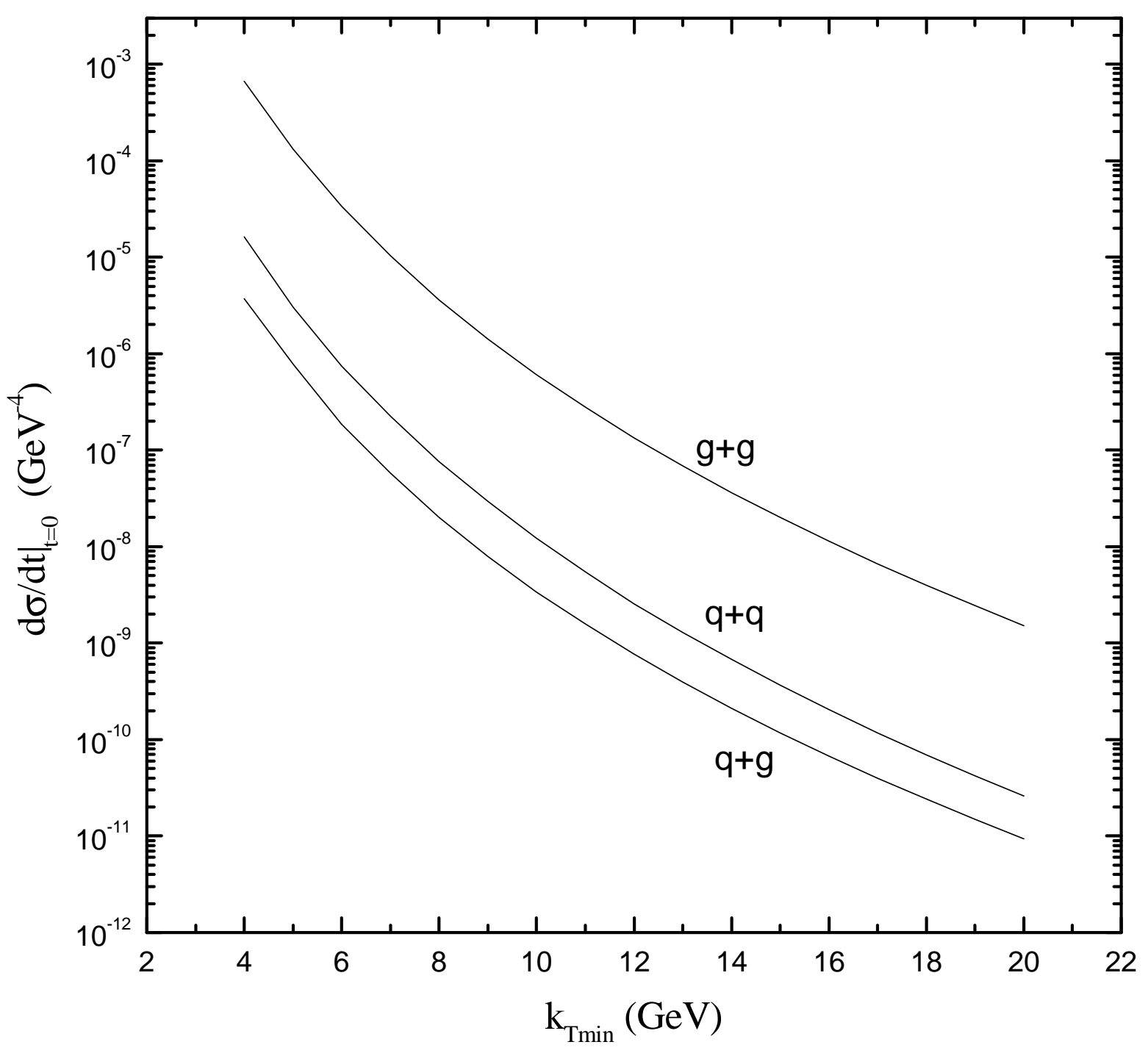

Fig.7 


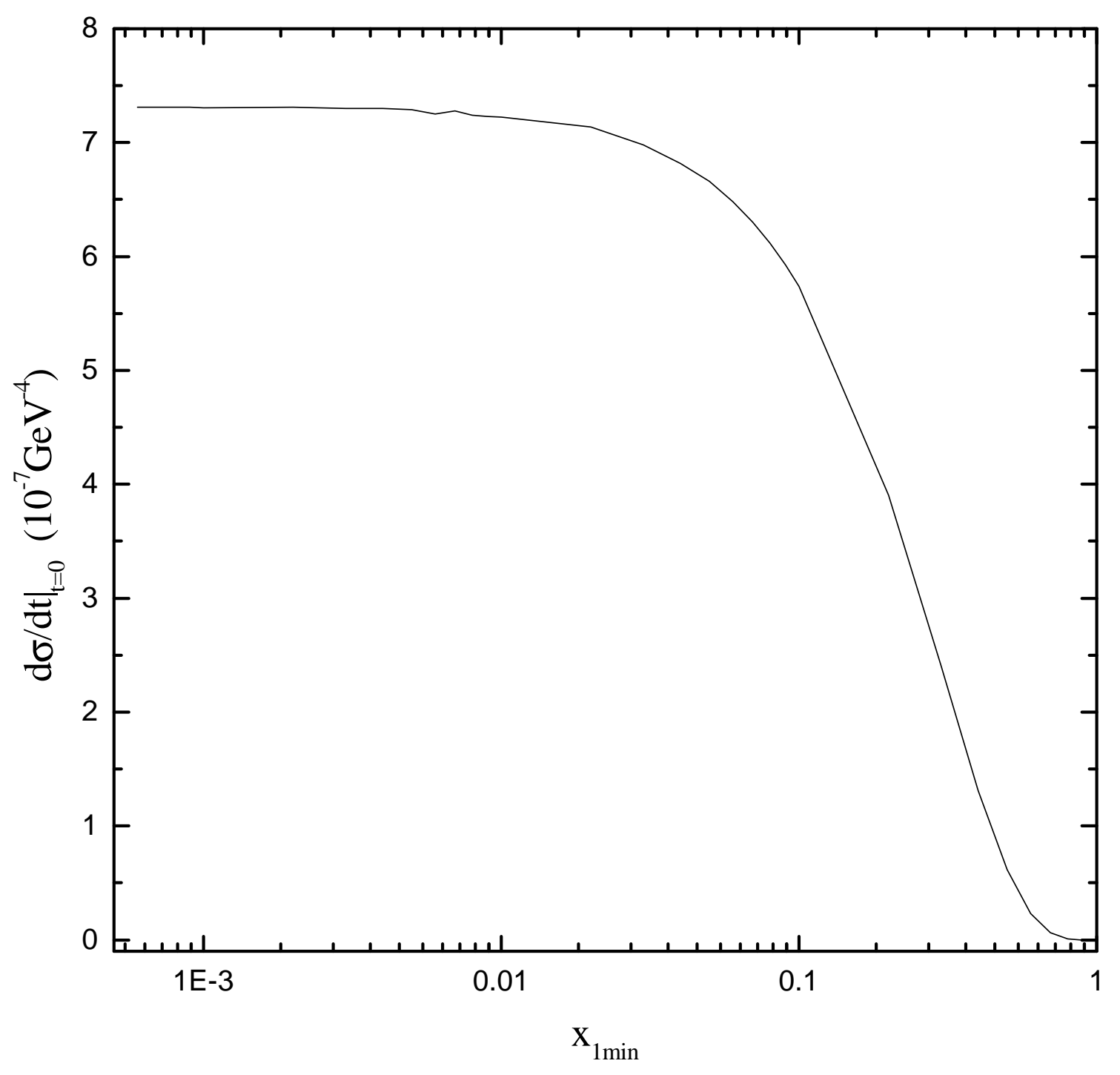

Fig.8(a) 


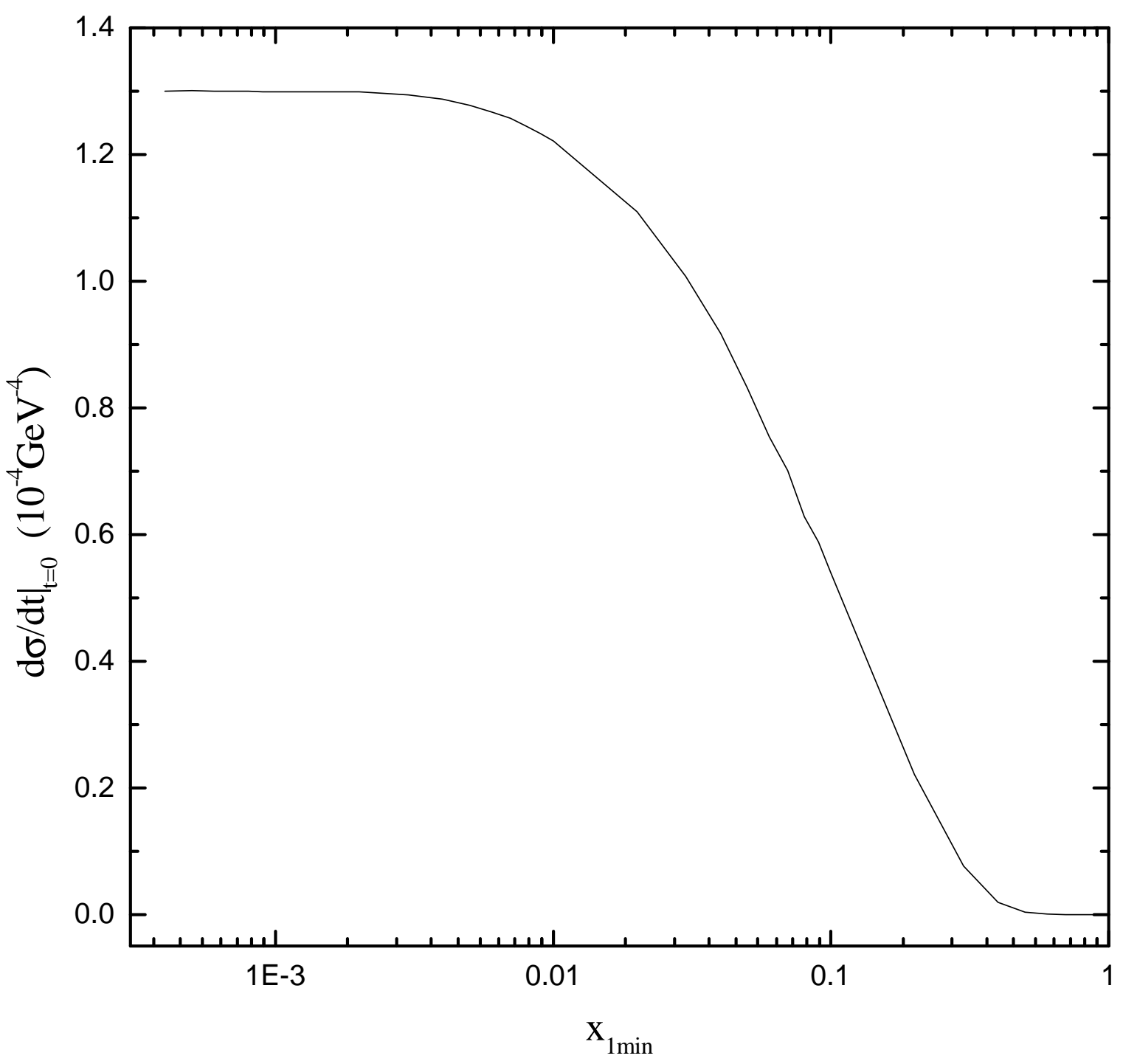

Fig.8(b) 